\title{
Global transcriptome response in Lactobacillus sakei during growth on ribose
}

\author{
Anette McLeod ${ }^{1,2^{*}}$, Lars Snipen ${ }^{2}$, Kristine Naterstad ${ }^{1}$ and Lars Axelsson ${ }^{1}$
}

\begin{abstract}
Background: Lactobacillus sakei is valuable in the fermentation of meat products and exhibits properties that allow for better preservation of meat and fish. On these substrates, glucose and ribose are the main carbon sources available for growth. We used a whole-genome microarray based on the genome sequence of $L$. sakei strain $23 \mathrm{~K}$ to investigate the global transcriptome response of three L. sakei strains when grown on ribose compared with glucose.

Results: The function of the common regulated genes was mostly related to carbohydrate metabolism and transport. Decreased transcription of genes encoding enzymes involved in glucose metabolism and the L-lactate dehydrogenase was observed, but most of the genes showing differential expression were up-regulated. Especially transcription of genes directly involved in ribose catabolism, the phosphoketolase pathway, and in alternative fates of pyruvate increased. Interestingly, the methylglyoxal synthase gene, which encodes an enzyme unique for $L$. sakei among lactobacilli, was up-regulated. Ribose catabolism seems closely linked with catabolism of nucleosides. The deoxyribonucleoside synthesis operon transcriptional regulator gene was strongly up-regulated, as well as two gene clusters involved in nucleoside catabolism. One of the clusters included a ribokinase gene. Moreover, hprK encoding the HPr kinase/phosphatase, which plays a major role in the regulation of carbon metabolism and sugar transport, was up-regulated, as were genes encoding the general PTS enzyme I and the mannose-specific enzyme II complex $\left(\mathrm{EII}^{\mathrm{man}}\right)$. Putative catabolite-responsive element (cre) sites were found in proximity to the promoter of several genes and operons affected by the change of carbon source. This could indicate regulation by a catabolite control protein A (CcpA)-mediated carbon catabolite repression (CCR) mechanism, possibly with the Ell ${ }^{\text {man }}$ being indirectly involved.

Conclusions: Our data shows that the ribose uptake and catabolic machinery in L. sakei is highly regulated at the transcription level. A global regulation mechanism seems to permit a fine tuning of the expression of enzymes that control efficient exploitation of available carbon sources.
\end{abstract}

\section{Background}

The Lactobacillus sakei species belongs to the lactic acid bacteria (LAB), a group of Gram-positive organisms with a low $\mathrm{G}+\mathrm{C}$ content which produce lactic acid as the main end product of carbohydrate fermentation. This trait has, throughout history, made LAB suitable for production of food. Acidification suppresses the growth and survival of undesirable spoilage bacteria and human pathogens. L. sakei is naturally associated with the meat and fish environment, and is important in the meat industry where it is used as starter culture for sausage fermentation $[1,2]$. The bacterium shows great potential as a protective

\footnotetext{
* Correspondence: anette.mcleod@nofima.no

${ }^{1}$ Nofima Mat AS, Norwegian Institute of Food, Fisheries and Aquaculture Research, Osloveien 1, Ås, NO-1430, Norway

Full list of author information is available at the end of the article
}

culture and biopreservative to extend storage life and ensure microbial safety of meat and fish products [3-6]. The genome sequence of $L$. sakei strain 23K has revealed a metabolic repertoire which reflects the bacterium's adaption to meat products and the ability to flexibly use meat components [7]. Only a few carbohydrates are available in meat and fish, and L. sakei can utilize mainly glucose and ribose for growth, a utilization biased in favour of glucose [7-9]. The species has been observed as a transient member of the human gastrointestinal tract (GIT) $[10,11]$, and ribose may be described as a commonly accessible carbon source in the gut environment [12]. Transit through the GIT of axenic mice gave mutant strains which grow faster on ribose compared with glucose [13].

Glucose is primarily transported and phosphorylated by the phosphoenolpyruvate (PEP)-dependent carbohydrate
C Biomed Central

(c) 2011 McLeod et al; licensee BioMed Central Ltd. This is an Open Access article distributed under the terms of the Creative Commons Attribution License (http://creativecommons.org/licenses/by/2.0), which permits unrestricted use, distribution, and reproduction in any medium, provided the original work is properly cited. 
phosphotransferase system (PTS). A phosphorylation cascade is driven from PEP through the general components enzyme I (EI) and the histidine protein ( $\mathrm{HPr}$ ), then via the mannose-specific enzyme II complex (EII ${ }^{\mathrm{man}}$ ) to the incoming sugar. Moreover, glucose is fermented through glycolysis leading to lactate $[7,8,14]$. Ribose transport and subsequent phosphorylation are induced by the ribose itself and mediated by a ribose transporter ( $\mathrm{RbsU}$ ), a Dribose pyranase (RbsD), and a ribokinase (RbsK) encoded by rbsUDK, respectively. These genes form an operon with $r b s R$ which encodes the local repressor RbsR $[15,16]$. The phosphoketolase pathway (PKP) is used for pentose fermentation ending with lactate and other end products $[8,17]$. L. sakei also has the ability to catabolize arginine, which is abundant in meat, and to catabolize the nucleosides inosine and adenine, a property which is uncommon among lactobacilli $[7,18]$.

By proteomics, we recently identified proteins involved in ribose catabolism and the PKP to be over-expressed during growth on ribose compared with glucose, while several glycolytic enzymes were less expressed. Moreover, also enzymes involved in pyruvate- and glycerol/glycerolipid metabolism were over-expressed on ribose [19]. Bacteria often use carbon catabolite repression (CCR) in order to control hierarchical utilization of different carbon sources. In low $\mathrm{G}+\mathrm{C}$ content Gram-positive bacteria, the dominant CCR pathway is mediated by the three main components: (1) catabolite control protein A (CcpA) transcriptional regulator; (2) the histidine protein ( $\mathrm{HPr}$ ); and (3) catabolite-responsive element (cre) DNA sites located in proximity to catabolic genes and operons, which are bound by CcpA [20-23]. The HPr protein has diverse regulatory functions in carbon metabolism depending on its phosphorylation state. In response to high throughput through glycolysis, the enzyme is phosphorylated at Ser46 by HPr kinase/phosphorylase (HPrK/P). This gives P-SerHPr which can bind to CcpA and convert it into its DNAbinding-competent conformation. However, when the concentration of glycolytic intermediates drop, the HPrK/ P dephosphorylates P-Ser-HPr [20,22-24]. Under low glucose concentrations, HPr is phosphorylated by E1 of the PTS at His15 to give P-His-HPr, which has a catalytic function in the PTS and regulatory functions by phosphorylation of catabolic enzymes and transcriptional regulators with a PTS regulation domain (PRD). Several P-EIIBs also phosphorylate different types of non-PTS proteins and regulate their activities [20-22]. Evidence for regulatory processes resembling glucose repression was shown both during lactose utilization [25] and catabolism of arginine $[26,27]$ in L. sakei. A cre site has been reported upstream of the rbs operon [28], thus CcpA could likely be acting on the $r b s$ operon as well as other catabolic genes and operons in this bacterium.
In the present study, we use a microarray representing the L. sakei $23 \mathrm{~K}$ genome and an additional set of sequenced $L$. sakei genes, to investigate the global transcriptome response of three L. sakei strains when grown on ribose compared with glucose. Moreover, we predict the frequency of cre sites presumed to be involved in CCR in the L. sakei $23 \mathrm{~K}$ genome sequence. Our objective was to identify differentially expressed genes between growth on the two sugars, and to increase the understanding of how the primary metabolism is regulated.

\section{Methods}

\section{Bacterial strains, media and growth conditions}

L. sakei $23 \mathrm{~K}$ is a plasmid-cured sausage isolate [29], and its complete genome sequence has been published [7]. L. sakei LS 25 is a commercial starter culture strain for salami sausage [30]. L. sakei MF1053 originates from fermented fish (Norwegian "rakfisk") [9]. The strains were maintained at $-80^{\circ} \mathrm{C}$ in MRS broth (Oxoid) supplemented with $20 \%$ glycerol. Growth experiments were performed in a defined medium for lactobacilli [31] supplemented with $0.5 \%$ glucose (DMLG) or $0.5 \%$ ribose + $0.02 \%$ glucose (DMLRg) as described previously [19]. Samples were extracted at three different days from independent DMLG and DMLRg cultures from each strain grown at $30^{\circ} \mathrm{C}$ to mid-exponential phase $\left(\mathrm{OD}_{600}=\right.$ 0.5-0.6) for a total of three sample sets (parallels).

\section{Microarrays}

The microarrays used have been described by Nyquist et al. [32], and a description is available at http://migale.jouy. inra.fr/sakei/Supplement.html/. 70-mer oligonucleotide probes representing the L. sakei strain $23 \mathrm{~K}$ genome and an additional set of sequenced $L$. sakei genes were printed in three copies onto epoxy glass slides (Corning).

\section{RNA extraction}

Total RNA extraction was performed using the RNeasy Protect Mini Prep Kit (Qiagen) as described by Rud et al. [33]. The concentration and purity of the total RNA was analysed using NanoDrop ND-1000 (NanoDrop Technologies), and the quality using Agilent 2100 Bioanalyzer (Agilent Technologies). Sample criteria for further use in the transcriptome analysis were $\mathrm{A}_{260} / \mathrm{A}_{280}$ ratio superior to 1.9 and 23S/16S RNA ratio superior to 1.6.

\section{cDNA synthesis, labeling, and hybridization}

cDNA was synthesized and labeled with the Fairplay III Microarray Labeling Kit (Stratagene, Agilent Technologies) as described previously [34]. After labeling, unincorporated dyes were removed from the samples using the QIAQuick PCR purification kit (Qiagen). The following prehybridization, hybridization, washing, and 
drying of the arrays were performed in a Tecan HS 400 Pro hybridization station (Tecan) as described by Nyquist et al. [32]. For studying the carbon effects, samples from DMLG and DMLRg were co-hybridized for each of the three strains. Separate hybridizations were performed for each strain on all three biological parallels. In order to remove potential biases associated with labelling and subsequent scanning, a replicate hybridization was performed for each strain for one of the three parallels, where the Cy3 and Cy5 dyes (GE Healthcare) used during cDNA synthesis were swapped. The hybridized arrays were scanned at wavelengths $532 \mathrm{~nm}$ (Cy3) and $635 \mathrm{~nm}$ (Cy5) with a Tecan scanner LS (Tecan). GenePix Pro 6.0 (Molecular Devices) was used for image analysis, and spots were excluded based on slide or morphology abnormalities.

\section{Microarray data analysis}

Downstream analysis was done by the Limma package http://www.bioconductor.org in the R computing environment http://www.r-project.org. Pre-processing and normalization followed a standard procedure using methods described by Smyth \& Speed [35], and testing for differential expressed genes were done by using a linear mixed model as described by Smyth [36]. A mixed-model approach was chosen to adequately describe betweenarray variation and still utilize probe-replicates (three replicates of each probe in each array). An empirical Bayes smoothing of gene-wise variances was conducted according to Smyth et al. [37], and for each gene the p-value was adjusted to control the false discovery rate (FDR), hence all p-values displayed are FDR-adjusted (often referred to as q-values in the literature).

\section{Validation of microarray data by qRT-PCR analysis}

The microarray results were validated on selected regulated genes for the LS 25 strain by quantitative real-time reverse transcriptase PCR (qRT-PCR) performed as described previously [38]. Primers and probes (Additional file 1, Table S3) were designed using Primer Express 3.0 (Applied Biosystems). Relative gene expression was calculated by the $\Delta C_{T}$ method, using the DNA gyrase subunit alpha gene $(g y r A)$ as the endogenous reference gene.

\section{Microarray accession numbers}

The microarray data have been deposited in the Array Express database http://www.ebi.ac.uk/arrayexpress/ under the accession numbers A-MEXP-1166 (array design) and E-MEXP-2892 (experiment).

\section{Sequence analysis}

A prediction of cre sites in the L. sakei $23 \mathrm{~K}$ genome sequence (GeneBank acc. no. CR936503.1), both strands, was performed based on the consensus sequence
TGWNANCGNTNWCA (W = A/T, N = A/T/G/C), confirmed in Gram-positive bacteria [39]. We made a search with the consensus sequence described by the regular expression T-G-[AT]-X-A-X-C-G-X-T-X-[AT]-C-A, allowing up to two mismatches in the conserved positions except for the two center position, highlighted in boldface. All computations were done in R http://www.r-project.org.

\section{Results and Discussion}

\section{Selection of $L$. sakei strains and growth conditions}

We have previously investigated $L$. sakei strain variation [9], and used proteomics to study the bacterium's primary metabolism [19], providing us with a basis for choosing strains with interesting differences for further studies. The starter culture strain LS 25 showed the fastest growth rates in a variety of media, and together with strain MF1053 from fish, it fermented the highest number of carbohydrates [9]. The LS 25 strain belongs to the L. sakei subsp. sakei, whereas the 23K and MF1053 strains belong to L. sakei subsp. carnosus $[9,19]$. By identification of differentially expressed proteins caused by the change of carbon source from glucose to ribose, LS 25 seemed to down-regulate the glycolytic pathway more efficiently than other strains during growth on ribose [19]. For these reasons, LS 25 and MF1053 were chosen in addition to $23 \mathrm{~K}$ for which the microarray is based on. Nyquist et al. [32] recently investigated the genomes of various $L$. sakei strains compared to the sequenced strain $23 \mathrm{~K}$ by comparative genome hybridization $(\mathrm{CGH})$ using the same microarray as in the present study. A large part of the $23 \mathrm{~K}$ genes belongs to a common gene pool invariant in the species, and the status for each gene on the array is known for all the three strains [32].

As glucose is the preferred sugar, L. sakei grows faster when glucose is utilized as the sole carbon source compared with ribose $[8,9,15]$. However, glucose stimulates ribose uptake and a possible co-metabolism of these two sugars present in meat and fish has been suggested, a possibility that give the organism an advantage in competition with other microbiota $[15,16,40]$. To obtain comparable 2 DE gels between samples issued from bacteria grown on the two carbohydrates in our recent proteomic analysis, growth on ribose was enhanced by adding small amounts of glucose [19]. For the present transcriptome analysis we therefore chose the same growth conditions.

\section{Global gene expression patterns}

A microarray representing the $L$. sakei $23 \mathrm{~K}$ genome and an additional set of sequenced $L$. sakei genes was used for studying the effect of carbon source on the transcriptome of L. sakei strains 23K, MF1053 and LS 25. Genes displaying a significant differential expression with a $\log _{2}$ ratio $>0.5$ or $<-0.5$ were classified into functional categories according to the L. sakei $23 \mathrm{~K}$ genome database 
http://migale.jouy.inra.fr/sakei/genome-server and are listed in Table 1 . The $23 \mathrm{~K}$ strain showed differential expression for 364 genes within these limits, MF1053 and LS 25 for 223 and 316 genes, respectively. Among these, 88,47 and 82 , respectively, were genes belonging to the category of genes of 'unknown' function. Eighty three genes, the expression of which varied depending on the carbon source, were common to the three strains, among which 52 were up-regulated and 31 down-regulated during growth on ribose (Figure 1). The function of these common regulated genes was mostly related to carbohydrate transport and metabolism (34 genes, Table 1$)$. The reliability of the microarray results was assessed by qRTPCR analysis using selected regulated genes in the LS 25 strain. As shown in Table S4 in the additional material (Additional file 1), the qRT-PCR results were in agreement with the data obtained by the microarrays.

Several of the up-regulated genes are located in operons, an organisation believed to provide the advantage of coordinated regulation. In addition, in order to discriminate genes induced by growth on ribose from those repressed by glucose (submitted to CCR mediated by CcpA), a search of the complete genome sequence of L. sakei $23 \mathrm{~K}$ [7] was undertaken, with the aim to identify putative cre sites. The search revealed 1962 hits, most of which did not have any biological significance considering their unsuitable location in relation to promoters. Relief of CcpAmediated CCR likely occur for many of the up-regulated genes in the category of carbohydrate transport and metabolism. Putative cre sites were identified in their promoter region, as well as for some genes involved in nucleoside and amino acid transport and metabolism (Table 2). In the other gene categories, the presences of putative cre sites were rare. With regard to gene product, the L. sakei genome shares high level of conservation with Lactobacillus plantarum [7], and high similarity of catabolic operon organization. The role of CcpA in CCR in L. plantarum has been established, and was shown to mediate regulation of the pox genes encoding pyruvate oxidases [41,42]. During growth on ribose, $L$. plantarum induces a similar set of genes as observed in the present study, and putative cre sites were identified in the upstream region of several genes involved [33].

\section{Ribose catabolism and PKP}

Confirming its major role in ribose transport and utilization in L. sakei, and in agreement with previous findings [16], our microarray data revealed a strong up-regulation (Table $1 ; \log _{2}=2.8-4.3$ ) of rbsUDK. The genes encoding an additional putative carbohydrate kinase belonging to the ribokinase family and a putative phosphoribosyl isomerase, $l s a 0254$ and $l s a 0255$, respectively, previously suggested to be involved in catabolism of ribose in L. sakei [7], were induced in all the strains (Table 1). Recent
CGH studies revealed that some L. sakei strains which were able to grow on ribose did not harbour the rbsK gene, whereas lsa0254 was present in all strains investigated [32]. This second ribokinase could therefore function as the main ribokinase in some L. sakei strains. The rbsK sequence could also differ considerably from that of $23 \mathrm{~K}$ in these strains. The PKP showed an obvious induction with an up-regulation (2.2-3.2) of the $x p k$ gene encoding the key enzyme xylulose-5-phosphate phosphoketolase (Xpk). This enzyme connects the upper part of the PKP to the lower part of glycolysis by converting xylulose-5-phosphate into glyceraldehyde-3-phosphate and acetyl-phosphate. Acetyl-phosphate is then converted to acetate and ATP by acetate kinase (Ack). Supporting our results, previous proteomic analysis showed an over-expression of RbsK, RbsD and Xpk during growth on ribose $[15,16,19]$. The induction of ribose transport and phosphorylation, and increased phosphoketolase and acetate kinase activities were previously observed during growth on ribose [15]. Three genes encoding Ack are present in the $23 \mathrm{~K}$ genome [7], as well as in MF1053 and LS 25 [32]. A preferential expression of different ack genes for the acetate kinase activity seem to exist. The ack 2 gene was up-regulated in all the strains, while ack1 was up-regulated and ack3 downregulated in $23 \mathrm{~K}$ and LS 25 (Table 1). An illustration of the metabolic pathways with genes affected by the change of carbon source from glucose to ribose in L. sakei is shown in Figure 2.

As a consequence of the pentose-induced PKP, genes involved in PKP-metabolism of glucose, such as gntZ, $g n t K$ and $z w f$, were down-regulated (Table 1, Figure 2). The glycolytic pathway was clearly repressed, supporting previous findings $[15,19]$. Among these genes were $p f k$ (0.5-1.1) encoding 6-phosphofructokinase (Pfk), and $f b a$ (0.7-1.1) coding for fructose-bisphosphate aldolase, both acting at the initial steps of glycolysis. In addition, gpm3 encoding one of the five phosphoglycerate mutases present in the $23 \mathrm{~K}$ genome, acting in the lower part of glycolysis, was also down-regulated (0.7-0.9). MF1053 down-regulated pyk (0.7) encoding pyruvate kinase (Pyk) that competes for PEP with the PTS (Figure 2). Its activity results in the production of pyruvate and ATP, and it is of major importance in glycolysis and energy production in the cell. MF1053 also showed a stronger downregulation of $p f k$ than the other strains (Table 1). Similar to several other lactobacilli, $p f k$ is transcribed together with pyk $[43,44]$, and in many microorganisms the glycolytic flux depends on the activity of the two enzymes encoded from this operon $[43,45]$. At the protein level, we previously observed both Pfk and Pyk expressed at a lower level for all the three strains [19], however this was not confirmed at the level of gene expression for $23 \mathrm{~K}$ and LS 25. We could also not confirm the lower 
Table 1 Genes with significant differential expression in three $L$. sakei strains grown on ribose compared with glucose, FDR adjusted $p$-value less than 0.01 and $\log _{2}$ of $>0.5$ or $<-0.5\left(\log _{2}\right.$ values $>1.0$ or $<-1.0$ are shown in bold)

\begin{tabular}{|c|c|c|}
\hline $\begin{array}{l}\text { Gene } \quad \text { Gene } \\
\text { locus }\end{array}$ & Description & 23K MF1053 \\
\hline
\end{tabular}

Carbohydrate transport and metabolism

\section{Transport/binding of carbohydrates}

\begin{tabular}{|c|c|c|c|c|c|}
\hline LSA0185* & galp & Galactose:cation symporter & 1.2 & & 1.7 \\
\hline LSA0200* & rbsU & Ribose transport protein & 2.8 & 3.5 & 4.3 \\
\hline LSA0353* & Isa0353 & Putative cellobiose-specific PTS, enzyme ॥B & 3.6 & 1.3 & 2.5 \\
\hline LSA0449* & manl & Mannose-specific PTS, enzyme IAB & 2.1 & 2.5 & 1.5 \\
\hline LSA0450* & $\operatorname{man} N$ & Mannose-specific PTS, enzyme IC & 1.9 & 2.0 & 1.4 \\
\hline LSA0451* & manM & Mannose-specific PTS, enzyme IID & 2.4 & 1.0 & 2.1 \\
\hline LSA0651* & glpF & Glycerol uptake facilitator protein, MIP family & 3.4 & 4.7 & 3.4 \\
\hline LSA1050* & fruA & Fructose-specific PTS, enzyme \|ABC & & & 0.9 \\
\hline LSA1204* & Isa1204 & Putative sugar transporter & & 1.1 & \\
\hline LSA1457* & Isa1457 & Putative cellobiose-specific PTS, enzyme ॥C & & 2.3 & \\
\hline LSA1462* & ptsl & PTS, enzyme I & 0.8 & 1.7 & 0.9 \\
\hline LSA1463* & ptsH & Phosphocarrier protein HPr (histidine protein) & & 1.2 & 0.9 \\
\hline LSA1533 & Isa1533 & Putative cellobiose-specific PTS, enzyme IIA & & 2.5 & 2.1 \\
\hline LSA1690 & Isa1690 & Putative cellobiose-specific PTS, enzyme ॥C & 0.9 & & \\
\hline LSA1792* & scrA & Sucrose-specific PTS, enzyme IIBCA & 0.8 & & 1.1 \\
\hline \multicolumn{6}{|c|}{ Metabolism of carbohydrates and related molecules } \\
\hline LSA0123* & Isa0123 & Putative sugar kinase, ROK family & 1.2 & & \\
\hline LSA0198 & ack1 & Acetate kinase (acetokinase) & 1.7 & & 1.3 \\
\hline LSA0254* & $1 s a 0254$ & Putative carbohydrate kinase & 2.4 & 0.8 & 1.8 \\
\hline LSA0292* & budC & Acetoin reductase (acetoin dehydrogenase) (meso-2,3-butanediol dehydrogenase) & 3.4 & 2.3 & 3.4 \\
\hline LSA0444 & Isa0444 & Putative malate dehydrogenase & 3.4 & $\mathrm{D}$ & 2.1 \\
\hline LSA0516 & hprk & Hpr kinase/phosphorylase & 2.0 & 1.6 & 1.2 \\
\hline LSA0664* & IOXL1N & L-lactate oxidase ( $\mathrm{N}$-terminal fragment), degenerate & 1.2 & & 0.7 \\
\hline LSA0665* & loxLI & L-lactate oxidase (central fragment), degenerate & 1.0 & & \\
\hline LSA0666* & IOXL1C & L-lactate oxidase (C-terminal fragment), degenerate & 1.0 & & \\
\hline LSA0974* & pflB & Formate C-acetyltransferase (pyruvate formate-lyase) (formate acetyltransferase) & 4.0 & & \\
\hline LSA0981 & ald $B$ & Acetolactate decarboxylase (alpha-acetolactate decarboxylase) & & 0.6 & 1.9 \\
\hline LSA0982 & als & Acetolactate synthase (alpha-acetolactate synthase) & & & 1.9 \\
\hline LSA0983 & Isa0983 & Putative aldose-1 epimerase & 0.6 & & \\
\hline LSA1032 & pyk & Pyruvate kinase & & -0.7 & \\
\hline LSA1080 & Isa1080 & Myo-inositol monophosphatase & 0.6 & & 0.8 \\
\hline$\underline{\text { LSA1082 }}$ & pdhD & Pyruvate dehydrogenase complex, E3 component, dihydrolipoamide dehydrogenase & 2.8 & 2.5 & 2.1 \\
\hline LSA1083 & pdhC & Puruvate dehydrogenase complex, E2 component, dihydrolipoamide acetyltransferase & 3.4 & 3.7 & 2.7 \\
\hline$\underline{L S A 1084}$ & $p d h B$ & Pyruvate dehydrogenase complex, E1 component, beta subunit & 3.2 & 3.3 & 2.2 \\
\hline LSA1085 & pdhA & Pyruvate dehydrogenase complex, E1 component, alpha subunit & 2.9 & 3.5 & 2.4 \\
\hline LSA1141* & $p p d K$ & Pyruvate phosphate dikinase & 1.0 & & 0.9 \\
\hline$\underline{\text { LSA1188* }}$ & pox1 & Pyruvate oxidase & 2.3 & 3.1 & 2.1 \\
\hline LSA1298 & ack2 & Acetate kinase (acetokinase) & 1.1 & 0.9 & 0.9 \\
\hline LSA1343* & eutD & Phosphate acetyltransferase (phosphotransacetylase) & 2.0 & 1.0 & 1.6 \\
\hline LSA1381 & Isa1381 & Putative acylphosphatase & -0.6 & -0.5 & \\
\hline LSA1399* & loxL2 & L-lactate oxidase & 3.4 & $U$ & \\
\hline LSA1630 & Isa1630 & Putative sugar kinase, ROK family & -0.6 & & -0.6 \\
\hline LSA1640* & nanA & $\mathrm{N}$-acetylneuraminate lyase & 2.0 & & $\mathrm{D}$ \\
\hline LSA1641* & nanE & $\mathrm{N}$-acylglucosamine/mannosamine-6-phosphate 2-epimerase & 0.9 & & $\mathrm{D}$ \\
\hline LSA1643* & Isa1643 & Putative sugar kinase, ROK family & 1.8 & & \\
\hline LSA1668 & ack3 & Acetate kinase (acetokinase) & -0.7 & & -1.1 \\
\hline LSA1830* & pox2 & Pyruvate oxidase & 0.7 & & \\
\hline
\end{tabular}


Table 1 Genes with significant differential expression in three $L$. sakei strains grown on ribose compared with glucose, FDR adjusted p-value less than 0.01 and $\log _{2}$ of $>0.5$ or $<-0.5\left(\log _{2}\right.$ values $>1.0$ or $<-1.0$ are shown in bold) (Continued)

\begin{tabular}{|c|c|c|c|c|c|}
\hline \multicolumn{6}{|c|}{ Intermediary metabolism } \\
\hline LSA0255* & Isa0255 & Putative phosphoribosyl isomerase & 2.0 & 1.0 & 1.6 \\
\hline \multicolumn{6}{|c|}{ Specific carbohydrate metabolic pathway } \\
\hline$\underline{\mathrm{LSA0201}}^{*}$ & $r b s D$ & D-ribose pyranase & 2.5 & 2.5 & 3.4 \\
\hline$\underline{\mathrm{LSA0202*}}$ & rbsk & Ribokinase & 3.0 & 3.9 & 4.3 \\
\hline$\underline{\mathrm{LSA0289*}}$ & xpk & Xylulose-5-phosphate phosphoketolase & 3.2 & 2.3 & 2.6 \\
\hline LSA0297 & gntZ & 6-phosphogluconate dehydrogenase & -1.2 & -0.9 & -1.7 \\
\hline LSA0298 & gntK & Gluconokinase & -0.8 & & \\
\hline LSA0381 & $z w f$ & Glucose-6-phosphate 1-dehydrogenase & -0.6 & -0.6 & -0.6 \\
\hline$\underline{\text { LSA0649* }}$ & glpK & Glycerol kinase & 3.4 & 4.8 & 2.1 \\
\hline LSA0650* & $g \mid p D$ & Glycerol-3-phosphate dehydrogenase & 2.3 & 2.2 & 2.0 \\
\hline LSA0764* & galk & Galactokinase & 1.1 & 0.7 & 1.8 \\
\hline LSA0765* & galE1 & UDP-glucose 4-epimerase & & & 1.2 \\
\hline LSA0766* & galt & Galactose-1-phosphate uridylyltransferase & 1.2 & 0.8 & 2.0 \\
\hline LSA0767* & galM & Aldose 1-epimerase (mutarotase) & 1.3 & & 2.0 \\
\hline LSA1146* & manA & Mannose-6-phosphate isomerase & 1.4 & 1.3 & 1.5 \\
\hline LSA1531 & Isa1531 & Putative beta-glucosidase & & 0.7 & 0.9 \\
\hline LSA1588 & nagA & $\mathrm{N}$-acetylglucosamine-6-phosphate deacetylase & 0.6 & & \\
\hline LSA1685 & rpiA & Ribose 5-phosphate epimerase (ribose 5-phosphate isomerase) & & 1.1 & 0.8 \\
\hline LSA1710* & lacM & Beta-galactosidase, small subunit (lactase, small subunit) & 3.3 & & 1.2 \\
\hline LSA1711* & lacL & Beta-galactosidase, large subunit (lactase, large subunit) & 3.0 & 1.5 & 1.7 \\
\hline LSA1790* & scrk & Fructokinase & & 1.0 & 1.1 \\
\hline LSA1791* & $\operatorname{dex} B$ & Glucan 1,6-alpha-glucosidase (dextran glucosidase) & & & 1.1 \\
\hline LSA1795 & melA & Alpha-galactosidase (melibiase) & & & -0.6 \\
\hline \multicolumn{6}{|c|}{ Glycolytic pathway } \\
\hline LSA0131 & gpm2 & Phosphoglycerate mutase & & 0.7 & \\
\hline$\underline{\text { LSA0206 }}$ & gpm3 & Phosphoglycerate mutase & -0.7 & -0.8 & -0.9 \\
\hline LSA0609* & gloAC & Lactoylglutathione lyase (C-terminal fragment), authentic frameshift & 1.1 & & 0.7 \\
\hline LSA0803 & gpm4 & Phosphoglycerate mutase & 0.5 & & 0.5 \\
\hline$\underline{\text { LSA1033 }}$ & pfk & 6-phosphofructokinase & -0.6 & -1.1 & -0.5 \\
\hline LSA1157 & mgsA & Methylglyoxal synthase & 2.3 & 1.4 & 1.7 \\
\hline LSA1179 & pgi & Glucose-6-phosphate isomerase & 0.5 & & \\
\hline$\underline{\text { LSA1527 }}$ & $f b a$ & Fructose-bisphosphate aldolase & -1.0 & -0.7 & -1.1 \\
\hline$\underline{\text { LSA1606 }}$ & IdhL & L-lactate dehydrogenase & -1.0 & -0.9 & -1.5 \\
\hline \multicolumn{6}{|c|}{ Nucleotide transport and metabolism } \\
\hline \multicolumn{6}{|c|}{ Transport/binding of nucleosides, nucleotides, purines and pyrimidines } \\
\hline LSA0013 & Isa0013 & Putative nucleobase:cation symporter & -0.9 & & -1.5 \\
\hline LSA0055 & Isa0055 & Putative thiamine/thiamine precursor:cation symporter & & & 1.6 \\
\hline LSA0064 & $15 a 0064$ & Putative nucleobase:cation symporter & & -0.8 & \\
\hline LSA0259 & Isa0259 & Pyrimidine-specific nucleoside symporter & 1.5 & & 1.3 \\
\hline LSA0798* & Isa0798 & Pyrimidine-specific nucleoside symporter & 3.5 & 2.2 & 1.7 \\
\hline LSA0799* & Isa0799 & Putative purine transport protein & 4.4 & 2.7 & 2.9 \\
\hline LSA1210 & Isa1210 & Putative cytosine:cation symporter (C-terminal fragment), authentic frameshift & -0.8 & & -0.6 \\
\hline LSA1211 & Isa1211 & Putative cytosine:cation symporter ( $\mathrm{N}$-terminal fragment), authentic frameshit & -1.1 & & -0.9 \\
\hline \multicolumn{6}{|c|}{ Metabolism of nucleotides and nucleic acids } \\
\hline LSA0010 & Isa0010 & Putative nucleotide-binding phosphoesterase & & & -0.6 \\
\hline LSA0023 & Isa0023 & Putative ribonucleotide reductase (Nrdl-like) & -0.5 & $\mathrm{D}$ & $\mathrm{D}$ \\
\hline LSA0063 & purA & Adenylosuccinate synthetase (IMP-aspartate ligase) & & -0.8 & \\
\hline LSA0139 & guaA & Guanosine monophosphate synthase (glutamine amidotransferase) & & -0.5 & -0.8 \\
\hline
\end{tabular}


Table 1 Genes with significant differential expression in three $L$. sakei strains grown on ribose compared with glucose, FDR adjusted $p$-value less than 0.01 and $\log _{2}$ of $>0.5$ or $<-0.5\left(\log _{2}\right.$ values $>1.0$ or $<-1.0$ are shown in bold) (Continued)

\begin{tabular}{|c|c|c|c|c|c|}
\hline LSA0252 & iunHl & Inosine-uridine preferring nucleoside hydrolase & 2.6 & 2.6 & 1.8 \\
\hline LSA0446 & pyrDB & Putative dihydroorotate oxidase, catalytic subunit & & & 0.9 \\
\hline LSA0489 & $15 a 0489$ & Putative metal-dependent phosphohydrolase precursor & 0.5 & & \\
\hline LSA0533* & iunH2 & Inosine-uridine preferring nucleoside hydrolase & 1.2 & & \\
\hline LSA0785 & $15 a 0785$ & Putative NCAIR mutase, PurE-related protein & -2.3 & & -1.3 \\
\hline LSA0795* & deoC & 2 Deoxyribose-5 phosphate aldolase & 4.0 & 2.1 & 2.2 \\
\hline LSA0796* & $\operatorname{deo} B$ & Phosphopentomutase (phosphodeoxyribomutase) & 5.5 & 4.1 & 3.2 \\
\hline LSA0797* & $\operatorname{deo} \mathrm{D}$ & Purine-nucleoside phosphorylase & 4.5 & 2.6 & 1.9 \\
\hline LSA0801* & $p d p$ & Pyrimidine-nucleoside phosphorylase & 1.8 & & \\
\hline LSA0940 & $n r d F$ & Ribonucleoside-diphosphate reductase, beta chain & & 1.0 & 0.6 \\
\hline LSA0941 & $n r d E$ & Ribonucleoside-diphosphate reductase, alpha chain & & 1.0 & 0.6 \\
\hline LSA0942 & $n r d H$ & Ribonucleotide reductase, $\mathrm{NrdH}$-redoxin & & 1.1 & \\
\hline LSA0950 & pyrR & $\begin{array}{l}\text { Bifunctional protein: uracil phosphoribosyltransferase and pyrimidine operon transcriptional } \\
\text { regulator }\end{array}$ & -0.6 & & \\
\hline LSA0993 & $r n h B$ & Ribonuclease HII (RNase HII) & & & 0.6 \\
\hline LSA1018 & $\mathrm{cmk}$ & Cytidylate kinase & & & 0.6 \\
\hline LSA1097 & Isa1097 & Putative ADP-ribose phosphorylase, NUDIX family & 0.5 & & \\
\hline LSA1352 & Isa1352 & Putative phosphomethylpyrimidine kinase & -0.8 & & \\
\hline LSA1651 & Isa1651 & Putative purine phosphoribosyltransferase, PRT family & & 0.8 & \\
\hline LSA1661 & Isa1661 & Putative nucleotide hydrolase, NUDIX family & & -0.5 & \\
\hline LSA1805 & $d g k$ & Deoxyguanosine kinase & -1.0 & & -0.8 \\
\hline
\end{tabular}

\section{Transcription}

Transcription regulation

$\begin{array}{llll}\text { LSA0130 Isa0130 Putative transcriptional regulator, Lacl family } & -0.6\end{array}$

LSA0132 Isa0132 Putative transcriptional regulator, MarR family $\quad-0.6$

LSA0161 Isa0161 Putative transcriptional regulator, ArsR family

LSA0186 Isa0186 Putative transcriptional regulator, LytR family

LSA0203 rbsR Ribose operon transcriptional regulator, Lacl family

1.7

LSA0217 Isa0217 Putative thiosulfate sulfurtransferase with a ArsR-HTH domain, rhodanese family

Putative transcriptional regulator, MerR family (N-terminal fragment), authentic frameshift

LSA0293 Isa0293 Putative DNA-binding protein, XRE family

LSA0356 rex1 Redox-sensing transcriptional repressor, Rex

LSA0603 cggR Glycolytic genes regulator

LSA0669 Isa0669 Putative transcription regulator, TetR family

LSA0783 Isa0783 Putative transcriptional regulator, Fnr/Crp Family

LSA0800 deoR Deoxyribonucleoside synthesis operon transcriptional regulator, GntR family

LSA0835 Isa0835 Putative DNA-binding protein, XRE family

LSA0848 rex Redox-sensing transcriptional repressor, Rex

LSA0972 Isa0972 Putative transcriptional regulator, LysR family

LSA1201 Isa1201 Putative transcriptional regulator, GntR family

LSA1322 glnR Glutamine synthetase transcriptional regulator, MerR family

LSA1351 Isa1351 Putative transcritional regulator with aminotransferase domain, GntR family

$\begin{array}{ccc} & & -0.6 \\ -0.8 & -0.5 & -0.6 \\ & -0.9 & -0.6 \\ & -0.6 & \\ & -0.6\end{array}$

LSA1434 Isa1434 Putative transcriptional regulator, DUF24 family (related to MarR/PadR families) 
Table 1 Genes with significant differential expression in three $L$. sakei strains grown on ribose compared with glucose, FDR adjusted $p$-value less than 0.01 and $\log _{2}$ of $>0.5$ or $<-0.5\left(\log _{2}\right.$ values $>1.0$ or $<-1.0$ are shown in bold) (Continued)

\begin{tabular}{llll}
\hline LSA1692 Isa1692 & Putative transcriptional regulator, GntR family & 0.7
\end{tabular}

CoEnzyme transport and metabolism

Metabolism of coenzymes and prostethic groups

LSA0041 PanE 2-dehydropantoate 2-reductase

LSA0057 thiE Thiamine-phosphate pyrophosphorylase (thiamine-phosphate synthase)

LSA0058 thiD Phosphomethylpyrimidine kinase (HMP-phosphate kinase)

LSA0059 thiM Hydroxyethylthiazole kinase (4-methyl-5-beta-hydroxyethylthiazole kinase)

LSA0183 Isa0183 Putative hydrolase, isochorismatase/nicotamidase family

LSA0840 Isa0840 Putative glutamate-cysteine ligase

1.0

$-0.7$

0.6

LSA0947 fhs Formate-tetrahydrofolate ligase (formyltetrahydrofolate synthetase)

LSA0980 Isa0980 Putative hydroxymethylpyrimidine/phosphomethylpyrimidine kinase, PfkB family

LSA1101 folK 2-amino-4-hydroxy-6-hydroxymethyldihydropteridine pyrophosphokinase

LSA1614 acpS

LSA1664 Isa1664
0.6

0.6

$-1.0$

1.6

$-0.9$

$-0.9$

$1.1 \quad 1.5$

\section{Energy production and conversion}

Membrane bioenergetics (ATP synthase)

$\begin{array}{lllr}\text { LSA1125 } & \text { atpC } & H(+) \text {-transporting two-sector ATPase (ATP synthase), epsilon subunit } & 0.6 \\ \text { LSA1126 } & \text { atpD } & H(+) \text {-transporting two-sector ATPase (ATP synthase), beta subunit } & 0.6 \\ \text { LSA1127 } & \text { atpG } & H(+) \text {-transporting two-sector ATPase (ATP synthase), gamma subunit } & 0.8 \\ \text { LSA1128 } & \text { atpA } & H(+) \text {-transporting two-sector ATPase (ATP synthase), alpha subunit } & 0.6 \\ \text { LSA1129 } & \text { atpH } & H(+) \text {-transporting two-sector ATPase (ATP synthase), delta subunit } & 0.6 \\ \text { LSA1130 } & \text { atpF } & H(+) \text {-transporting two-sector ATPase (ATP synthase), B subunit } & 0.5 \\ \text { LSA1131 } & \text { atpE } & H(+) \text {-transporting two-sector ATPase (ATP synthase), C subunit } & 0.7\end{array}$

Inorganic ion transport and metabolism

\section{Transport/binding of inorganic ions}

$\begin{array}{lll}\text { LSA0029 } & \text { Isa0029 } & \text { Putative ion Mg(2+)/Co(2+) transport protein, hemolysinC-family } \\ \text { LSA0134 } & \text { Isa0134 } & \text { Putative Na(+)/H(+) antiporter } \\ \text { LSA0180 } & \text { mtSC } & \text { Manganese ABC transporter, ATP-binding subunit } \\ \text { LSA0181 } & \text { mtsB } & \text { Manganese ABC transporter, membrane-spanning subunit } \\ \text { LSA0182 } & \text { mtsA } & \text { Manganese ABC transporter, substrate-binding lipoprotein precursor } \\ \text { LSA0246 } & \text { mntH1 } & \text { Mn(2+)/Fe(2+) transport protein } \\ \text { LSA0283 } & \text { Isa0283 } & \text { Putative zinc/iron ABC transporter, ATP-binding subunit } \\ \text { LSA0284 } & \text { Isa0284 } & \text { Putative zinc/iron ABC transporter, membrane-spanning subunit } \\ \text { LSA0399 } & \text { Isa0399 } & \text { Iron(III)-compound ABC transporter, substrate-binding lipoprotein precursor } \\ \text { LSA0400 } & \text { Isa0400 } & \text { Iron(III)-compound ABC transporter, ATP-binding subunit } \\ \text { LSA0401 } & \text { Isa0401 } & \text { Iron(III)-compound ABC transporter, membrane-spanning subunit } \\ \text { LSA0402 } & \text { Isa0402 } & \text { Iron(III)-compound ABC transporter, membrane-spanning subunit } \\ \text { LSA0503 } & \text { pstC } & \text { Phosphate ABC transporter, membrane-spanning subunit } \\ \text { LSA0504 } & \text { pstA } & \text { Phosphate ABC transporter, membrane-spanning subunit } \\ \text { LSA0781 } & \text { Isa0781 } & \text { Putative cobalt ABC transporter, membrane-spanning/permease subunit } \\ \text { LSA0782 } & \text { Isa0782 } & \text { Putative cobalt ABC transporter, membrane-spanning/permease subunit } \\ \text { LSA1166 } & \text { Isa1166 } & \text { Putative potassium transport protein } \\ \text { LSA1440 } & \text { cutC } & \text { Copper homeostasis protein, CutC family } \\ \text { LSA1460 } & \text { atkB } & \text { Copper-transporting P-type ATPase } \\ \text { LSA1638 } & \text { Isa1638 } & \text { Putative large conductance mechanosensitive channel } \\ \text { LSA1645 } & \text { Isa1645 } & \text { Putative Na(+)/(+) antiporter } \\ \text { LSA1699 } & \text { mntH2 } & \text { Mn(2+)/Fe(2+) transport protein }\end{array}$


Table 1 Genes with significant differential expression in three $L$. sakei strains grown on ribose compared with glucose, FDR adjusted $p$-value less than 0.01 and $\log _{2}$ of $>0.5$ or $<-0.5\left(\log _{2}\right.$ values $>1.0$ or $<-1.0$ are shown in bold) (Continued)

\begin{tabular}{llll}
\hline LSA1703 & Isa1703 & Putative Na(+)/H(+) antiporter & $\mathbf{- 1 . 2}$ \\
LSA1704 & Isa1704 & Putative calcium-transporting P-type ATPase & -0.8 \\
LSA1735 & Isa1735 & Putative cobalt ABC transporter, membrane-spanning subunit & -0.6 \\
LSA1736 & Isa1736 & Putative cobalt ABC transporter, ATP-binding subunit & -0.6 \\
LSA1737 & Isa1737 & Putative cobalt ABC transporter, ATP-binding subunit & -0.7 \\
LSA1838 & Isa1838 & Putative metal ion ABC transporter, membrane-spanning subunit & -0.5 \\
LSA1839 & Isa1839 & Putative metal ion ABC transporter, substrate-binding lipoprotein precursor & -0.6
\end{tabular}

\section{Amino acid transport and metabolism}

\section{Transport/binding of amino acids}

\begin{tabular}{|c|c|c|}
\hline LSA0125 & $15 a 0125$ & Putative amino acid/polyamine transport protein \\
\hline LSA0189 & $15 a 0189$ & Putative amino acid/polyamine transport protein \\
\hline LSA0311 & Isa0311 & Putative glutamate/aspartate:cation symporter \\
\hline LSA1037 & Isa1037 & Putative amino acid/polyamine transport protein \\
\hline LSA1219 & Isa1219 & Putative cationic amino acid transport protein \\
\hline LSA1415 & Isa1415 & Putative amino acid/polyamine transport protein \\
\hline LSA1424 & Isa1424 & Putative L-aspartate transport protein \\
\hline LSA1435 & Isa1435 & Putative amino acid:H(+) symporter \\
\hline LSA1496 & Isa1496 & Putative glutamine/glutamate ABC transporter, ATP-binding subunit \\
\hline LSA1497 & Isa1497 & $\begin{array}{l}\text { Putative glutamine/glutamate } A B C \text { transporter, membrane-spanning/substrate-binding subunit } \\
\text { precursor }\end{array}$ \\
\hline
\end{tabular}

Transport/binding of proteins/peptides

\begin{tabular}{|c|c|c|}
\hline LSA0702 & oppA & Oligopeptide ABC transporter, substrate-binding lipoprotein precursor \\
\hline LSA0703 & $O p p B$ & Oligopeptide ABC transporter, membrane-spanning subunit \\
\hline LSA0704 & oppC & Oligopeptide ABC transporter, membrane-spanning subunit \\
\hline LSA0705 & oppD & Oligopeptide ABC transporter, ATP-binding subunit \\
\hline LSA0706 & oppF & Oligopeptide ABC transporter, ATP-binding subunit \\
\hline \multicolumn{3}{|c|}{ Protein fate } \\
\hline LSA0053 & рерО & Endopeptidase $\mathrm{O}$ \\
\hline LSA0133 & pepR & Prolyl aminopeptidase \\
\hline LSA0226 & $\operatorname{pep} N$ & Aminopeptidase N (lysyl-aminopeptidase-alanyl aminopeptidase) \\
\hline LSA0285 & pepF1 & Oligoendopeptidase F1 \\
\hline LSA0320 & pepD3 & Dipeptidase D-type (U34 family) \\
\hline LSA0424 & pepV & Xaa-His dipeptidase V (carnosinase) \\
\hline LSA0643 & pepX & X-Prolyl dipeptidyl-aminopeptidase \\
\hline LSA0888 & рерт & Tripeptide aminopeptidase $T$ \\
\hline LSA1522 & peps & Aminopeptidase S \\
\hline LSA1686 & pepCiN & Cysteine aminopeptidase C1 (bleomycin hydrolase) ( $\mathrm{N}$-terminal fragment), authentic frameshift \\
\hline LSA1688 & pepC2 & Cysteine aminopeptidase C2 (bleomycin hydrolase) \\
\hline LSA1689 & Isa1689 & Putative peptidase M20 family \\
\hline \multicolumn{3}{|c|}{ Metabolism of amino acids and related molecules } \\
\hline LSA0220_C & dapE & Succinyl-diaminopimelate desuccinylase \\
\hline LSA0316 & $\operatorname{sdh} B$ & L-serine dehydratase, beta subunit (L-serine deaminase) \\
\hline LSA0370* & $\operatorname{arcA}$ & Arginine deiminase (arginine dihydrolase) \\
\hline LSA0372* & $\operatorname{arcc}$ & Carbamate kinase \\
\hline LSA0463 & $15 a 0463$ & Putative 2-hydroxyacid dehydrogenase \\
\hline LSA0509 & kbl & 2-amino-3-ketobutyrate coenzyme A ligase (glycine acetyltransferase) \\
\hline LSA0510 & $15 a 0510$ & L-threonine dehydrogenase ( $\mathrm{N}$-terminal fragment), authentic frameshift \\
\hline LSA0572* & $t d c B$ & Threonine deaminase (threonine ammonia-lyase, threonine dehydratase, IIvA homolog) \\
\hline LSA0922 & serA & D-3-phosphoglycerate dehydrogenase \\
\hline LSA1134 & glyA & Glycine/Serine hydroxymethyltransferase \\
\hline
\end{tabular}


Table 1 Genes with significant differential expression in three $L$. sakei strains grown on ribose compared with glucose, FDR adjusted $p$-value less than 0.01 and $\log _{2}$ of $>0.5$ or $<-0.5\left(\log _{2}\right.$ values $>1.0$ or $<-1.0$ are shown in bold) (Continued)

\begin{tabular}{lllcc}
\hline LSA1321 & glnA & Glutamate-ammonia ligase (glutamine synthetase) & -1.3 & -1.0 \\
LSA1484 & mvaS & Hydroxymethylglutaryl-CoA synthase & -0.7 & -0.6 \\
LSA1693 & asnA2 & L-asparaginase & -0.7 \\
\end{tabular}

Lipid transport and metabolism

Metabolism of lipids

\begin{tabular}{|c|c|c|c|c|}
\hline LSA0045 & $c f a$ & Cyclopropane-fatty-acyl-phospholipid synthase & -1.3 & -1.4 \\
\hline LSA0644 & $15 a 0644$ & Putative acyl-CoA thioester hydrolase & 0.6 & \\
\hline LSA0812 & fabZ1 & (3R)-hydroxymyristoyl-[acyl-carrier protein] dehydratase & & -0.7 \\
\hline LSA0813 & $\mathrm{fabH}$ & 3-oxoacyl-[acyl carrier protein] synthetase III & & \\
\hline LSA0814 & $\operatorname{acp} P$ & Acyl carrier protein & & \\
\hline LSA0815 & $f a b D$ & Malonyl-CoA:ACP transacylase & & -0.7 \\
\hline LSA0816 & $f a b G$ & 3-oxoacyl-acyl carrier protein reductase & & -0.7 \\
\hline LSA0817 & $f a b F$ & 3-oxoacyl-[acyl carrier protein] synthetase II & & -0.7 \\
\hline LSA0819 & $f a b Z$ & (3R)-hydroxymyristoyl-[acyl carrier proetin] dehydratase & & \\
\hline LSA0820 & $\operatorname{accc}$ & Acetyl-CoA carboxylase (biotin carbooxylase subunit) & & -0.7 \\
\hline LSA0821 & $a c c D$ & Acetyl-CoA carboxylase (carboxyl transferase beta subunit) & & \\
\hline LSA0822 & $\operatorname{acc} A$ & Acetyl-CoA carboxylase (carboxyl transferase alpha subunit) & & \\
\hline LSA0823 & fabl & Enoyl [acyl carrier protein] reductase & & \\
\hline LSA0891 & Isa0891 & Putative lipase/esterase & 1.2 & \\
\hline LSA1485 & mvaA & Hydroxymethylglutaryl-CoA reductase & -0.5 & \\
\hline LSA1493 & Isa1493 & Putative diacylglycerol kinase & -0.6 & -0.9 \\
\hline LSA1652 & ipk & 4-diphosphocytidyl-2-C-methyl-D-erythritol kinase & -0.6 & \\
\hline
\end{tabular}

Secondary metabolites transport and metabolism

Transport/binding proteins and lipoproteins

\begin{tabular}{|c|c|c|c|c|c|}
\hline LSA0046 & Isa0046 & Putative transport protein & -1.0 & -0.6 & -1.3 \\
\hline LSA0089 & $15 a 0089$ & Putative drug transport protein & -2.1 & -0.9 & -0.8 \\
\hline LSA0094 & $15 a 0094$ & Putative transport protein, Major Facilitator Super (MFS) family transporter & -0.7 & & -0.7 \\
\hline LSA0095 & Isa0095 & Putative transport protein & 1.3 & 0.5 & \\
\hline LSA0128 & $15 a 0128$ & Putative antimicrobial peptide $A B C$ exporter, membrane-spanning/permease subunit & & & -0.5 \\
\hline LSA0187 & Isa0187 & Putative drug-resistance ABC transporter, two ATP-binding subunits & & 0.7 & \\
\hline LSA0219_b & Isa0219_b & Putative cyanate transport protein & -0.6 & & \\
\hline LSA0232 & ImrA & Multidrug ABC exporter, ATP-binding and membrane-spanning/permease subunits & -0.7 & & -0.7 \\
\hline LSA0270 & $15 a 0270$ & Putative multidrug ABC exporter, membrane-spanning/permease subunit & -0.7 & & \\
\hline LSA0271 & Isa0271 & Putative multidrug ABC exporter, ATP-binding subunit & -0.7 & & -0.6 \\
\hline LSA0272 & 1sa0272 & Putative multidrug ABC exporter, ATP-binding and membrane-spanning/permease subunits & -0.6 & & -0.6 \\
\hline LSA0308 & $15 a 0308$ & Putative drug:H(+) antiporter & & & -0.7 \\
\hline LSA0376 & $15 a 0376$ & Putative transport protein & 0.7 & & \\
\hline LSA0420 & $15 a 0420$ & Putative drug: $\mathrm{H}(+)$ antiporter (N-terminal fragment), authentic frameshift & -0.8 & & -1.1 \\
\hline LSA0469 & $15 a 0469$ & Putative drug:H(+) antiporter & -0.6 & & -0.5 \\
\hline LSA0788 & $15 a 0788$ & Putative facilitator protein, MIP family & -2.6 & & \\
\hline LSA0936 & Isa0936 & Putative drug $A B C$ exporter, membrane-spanning/permease subunit & 1.1 & & \\
\hline LSA0937 & Isa0937 & Putative drug $A B C$ exporter, membrane-spanning/permease subunit & 1.3 & & \\
\hline LSA0938 & 1500938 & Putative drug ABC exporter, ATP-binding subunit & 1.2 & & \\
\hline LSA0963 & Isa0963 & Integral membrane protein, hemolysin III related & & & \\
\hline LSA1088 & Isa1088 & Putative multidrug ABC exporter, ATP-binding and membrane-spanning/permease subunits & 0.5 & & \\
\hline LSA1261 & Isa1261 & Putative autotransport protein & 0.5 & & \\
\hline LSA1340 & $1 s a 1340$ & Putative transport protein & & -0.7 & \\
\hline LSA1366 & Isa1366 & Putative $A B C$ exporter, ATP-binding subunit & -0.8 & & -1.0 \\
\hline
\end{tabular}


Table 1 Genes with significant differential expression in three $L$. sakei strains grown on ribose compared with glucose, FDR adjusted p-value less than 0.01 and $\log _{2}$ of $>0.5$ or $<-0.5\left(\log _{2}\right.$ values $>1.0$ or $<-1.0$ are shown in bold) (Continued)

\begin{tabular}{lllcl}
\hline LSA1367 & Isa1367 & Putative ABC exporter, membrane-spanning/permease subunit & -0.8 & -0.5 \\
LSA1420 & Isa1417 & Putative lipase/esterase & -0.8 \\
LSA1621 & Isa1621 & Putative drug:H(+) antiporter & $\mathbf{- 1 . 1}$ \\
LSA1642 & Isa1642 & Putative Solute:Na(+) symporter & $\mathbf{- 1 . 1}$ \\
LSA1872 & Isa1872 & Putative drug:H(+) antiporter & $\mathbf{3 . 4}$ & $\mathbf{1 . 8}$ \\
LSA1878 & Isa1878 & Putative drug resistance ABC transporter, two ATP-binding subunits & $\mathbf{D}$ \\
Detoxification & & -0.6 \\
LSA0772 & Isa0772 & Hypothetical protein (TelA, telluric resistance family) & $\mathbf{1 . 0}$ \\
LSA1317 & Isa1317 & Putative chromate reductase & 0.6 \\
LSA1450 & Isa1450 & Putative metal-dependent hydrolase (beta-lactamase family III) & -0.7 \\
LSA1776 & Isa1776 & Putative 4-carboxymuconolactone decarboxylase & 0.7 \\
\end{tabular}

Translation, ribosomal structure and biogenesis

Translation initiation

LSA1135 Isa1135 Putative translation factor, Sua5 family

Translation elongation

LSA0251 efp1 Elongation factor P (EF-P)

LSA1063 tuf

Elongation factor Tu (EF-Tu)

Ribosomal proteins

\begin{tabular}{|c|c|c|}
\hline LSA0011 & rpll & 50S Ribosomal protein L9 \\
\hline LSA0266 & $r p s N$ & $30 S$ ribosomal protein S14 \\
\hline LSA0494 & Isa0494 & 30 sibosomal interface protein S30EA \\
\hline A0696 & rpmB & $50 S$ ribosomal protein L28 \\
\hline SA1017 & $r p s A$ & 30 Sibosomal protein S1 \\
\hline LSA1333 & rpmG & $50 S$ ribosomal protein L33 \\
\hline LSA1666 & $r p / L$ & $50 S$ ribosomal protein $\mathrm{L} 7 / \mathrm{L} 12$ \\
\hline LSA1676 & rpmG2 & $50 S$ ribosomal protein L33 \\
\hline LSA1750 & $r p l F$ & $50 S$ ribosomal protein L6 \\
\hline LSA1755 & $r p s Q$ & $30 S$ ribosomal protein $\mathrm{S} 17$ \\
\hline LSA1761 & $r p / B$ & $50 S$ ribosomal protein $\mathrm{L} 2$ \\
\hline LSA1765 & rps」 & $30 S$ ribosomal protein $\mathrm{S} 10$ \\
\hline \multicolumn{3}{|c|}{ Protein synthesis } \\
\hline LSA0377 & tgt & Queuine tRNA-ribosyltransferase \\
\hline LSA1546 & gatB & Glutamyl-tRNA amidotransferase, subunit B \\
\hline LSA1547 & gatA & Glutamyl-tRNA amidotransferase, subunit A \\
\hline \multicolumn{3}{|c|}{ RNA restriction and modification } \\
\hline LSA0437 & $15 a 0437$ & Hypothetical protein with an RNA-binding domain \\
\hline LSA0443 & Isa0443 & Putative single-stranded mRNA endoribonuclease \\
\hline LSA0738 & $d t d$ & D-tyrosyl-tRNA(tyr) deacylase \\
\hline LSA0794 & $\operatorname{trm} U$ & tRNA (5-methylaminomethyl-2-thiouridylate)-methyltransferase \\
\hline LSA1534 & Isa1534 & Putative ATP-dependent RNA helicase \\
\hline LSA1615 & Isa1615 & Putative ATP-dependent RNA helicase \\
\hline LSA1723 & $\operatorname{truA}$ & tRNA pseudouridylate synthase A (pseudouridylate synthase I) \\
\hline LSA1880 & trme & tRNA modification GTPase trmE \\
\hline
\end{tabular}

Aminoacyl-tRNA synthetases

$\begin{array}{lll}\text { LSA0880 } & \text { glyQ } & \text { Glycyl-tRNA synthetase, alpha subunit } \\ \text { LSA0881 } & \text { glyS } & \text { Glycyl-tRNA synthetase, beta subunit } \\ \text { LSA1400 } & \text { thrs } & \text { Threonyl-tRNA synthetase } \\ \text { LSA1681 } & \text { cysS } & \text { Cysteinyl-tRNA synthetase }\end{array}$


Table 1 Genes with significant differential expression in three $L$. sakei strains grown on ribose compared with glucose, FDR adjusted p-value less than 0.01 and $\log _{2}$ of $>0.5$ or $<-0.5\left(\log _{2}\right.$ values $>1.0$ or $<-1.0$ are shown in bold) (Continued)

\begin{tabular}{|c|c|c|c|c|c|}
\hline \multicolumn{6}{|c|}{ DNA replication, recombination and repair } \\
\hline \multicolumn{6}{|c|}{ DNA replication } \\
\hline LSA0221 & Isa0221 & Putative transcriptional regulator, LysR family (C-terminal fragment), degenerate & -0.8 & -0.9 & -1.1 \\
\hline LSA0976 & parE & Topoisomerase IV, subunit B & & 0.5 & \\
\hline \multicolumn{6}{|c|}{ Transposon and IS } \\
\hline LSA1152_a & $\begin{array}{l}\text { tnpA3- } \\
\text { ISLsa1 }\end{array}$ & Transposase of ISLsa1 (IS30 family) & -0.6 & & \\
\hline \multicolumn{6}{|c|}{ Phage-related function } \\
\hline LSA1292 & Isa1292 & Putative prophage protein & 0.6 & & \\
\hline LSA1788 & Isa1788 & Putative phage-related 1,4-beta- $\mathrm{N}$-acetyl muramidase (cell wall hydrolase) & -1.0 & $\mathrm{D}$ & D \\
\hline \multicolumn{6}{|c|}{ DNA recombination and repair } \\
\hline LSA0076 & Isa0076 & Putative DNA invertase (plasmidic resolvase) & -1.1 & -1.5 & -1.4 \\
\hline LSA0366 & ruvA & Holliday junction DNA helicase RuvA & & & -0.5 \\
\hline LSA0382 & $\operatorname{din} P$ & DNA-damage-inducible protein $P$ & -0.5 & & \\
\hline LSA0487 & recA & DNA recombinase $A$ & -0.8 & & -1.1 \\
\hline LSA0523 & $u v r B$ & Excinuclease $A B C$, subunit $B$ & -0.7 & & -0.5 \\
\hline LSA0524 & UvrAl & Excinuclease $A B C$, subunit $A$ & -1.2 & & -0.7 \\
\hline LSA0910 & rexAN & ATP-dependent exonuclease, subunit A ( $\mathrm{N}$-terminal fragment), authentic frameshift & 0.6 & & \\
\hline LSA0911 & rexAC & ATP-dependent exonuclease, subunit A (C-terminal fragment), authentic frameshift & 0.7 & & \\
\hline LSA0912 & $15 a 0912$ & Putative ATP-dependent helicase, DinG family & 0.6 & & 0.8 \\
\hline LSA1162 & Isa1162 & DNA-repair protein (SOS response UmuC-like protein) & & 0.8 & -0.6 \\
\hline LSA1405 & fpg & Formamidopyrimidine-DNA glycosylase & -0.5 & -0.6 & -0.6 \\
\hline LSA1477 & rec $X$ & Putative regulatory protein, $\operatorname{Rec} X$ family & -0.6 & & \\
\hline LSA1843 & ogt & Methylated-DNA-protein-cysteine S-methyltransferase & -0.6 & & \\
\hline \multicolumn{6}{|c|}{ DNA restriction and modification } \\
\hline LSA0143 & Isa0143 & Putative adenine-specific DNA methyltransferase & -0.7 & $\mathrm{D}$ & D \\
\hline LSA0921 & Isa0921 & Putative adenine-specific DNA methyltransferase & 0.8 & & \\
\hline LSA1299 & Isa1299 & Putative adenine-specific DNA methyltransferase & 0.9 & 0.7 & 1.2 \\
\hline \multicolumn{6}{|c|}{ Information pathways } \\
\hline LSA0326 & Isa0326 & Putative DNA helicase & & -0.6 & U \\
\hline \multicolumn{6}{|c|}{ DNA packaging and segregation } \\
\hline LSA0135 & Isa0135 & Hypothetical integral membrane protein, similar to $\mathrm{CcrB}$ & & & -0.6 \\
\hline LSA1015 & hbsU & Histone-like DNA-binding protein $\mathrm{HU}$ & 1.0 & & 0.9 \\
\hline
\end{tabular}

Cell division and chromosome partitioning

\section{Cell division}

$\begin{array}{lllr}\text { LSA0755 } & \text { divIVA } & \text { Cell-division initiation protein (septum placement) } & 0.5 \\ \text { LSA0845 } & \text { Isa0845 } & \text { Putative negative regulator of septum ring formation } & 0.6 \\ \text { LSA1118 } & \text { Isa1118 } & \text { Rod-shape determining protein } & 0.7 \\ \text { LSA1597 } & \text { ftsH } & \text { ATP-dependent zinc metalloendopeptidase FtsH (cell division protein FtsH) } & 0.5 \\ \text { LSA1879 } & \text { gidA } & \text { Cell division protein GidA } & -0.6\end{array}$

Cell envelope biogenesis, outer membrane

Cell wall

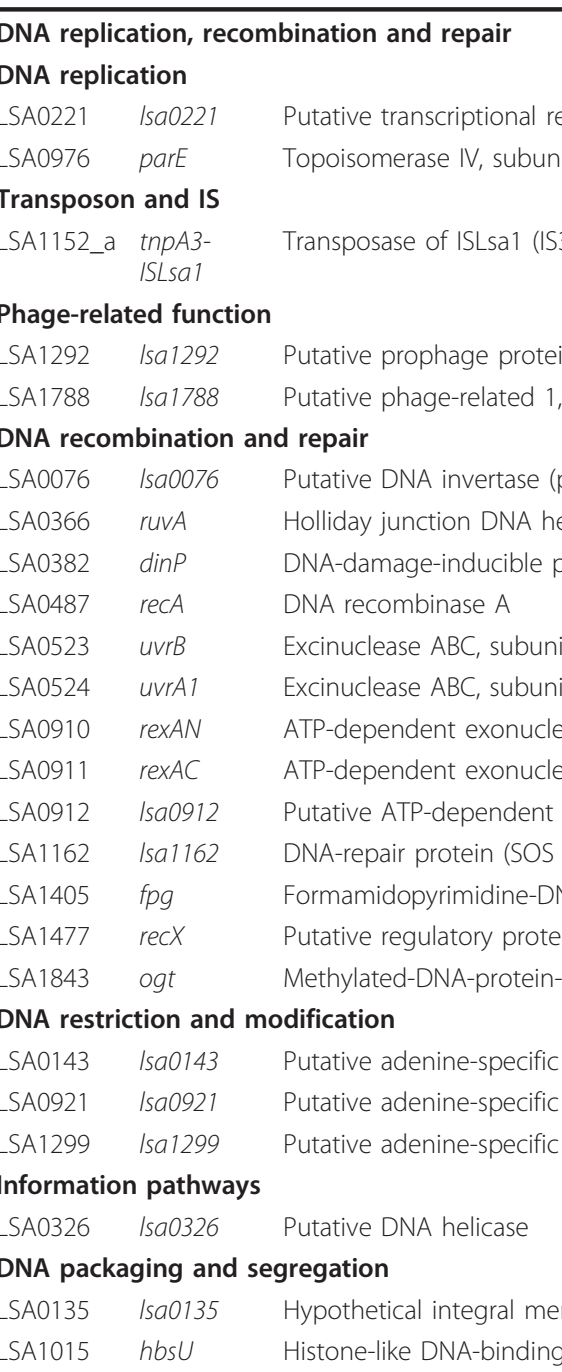


Table 1 Genes with significant differential expression in three $L$. sakei strains grown on ribose compared with glucose, FDR adjusted p-value less than 0.01 and $\log _{2}$ of $>0.5$ or $<-0.5\left(\log _{2}\right.$ values $>1.0$ or $<-1.0$ are shown in bold) (Continued)

\begin{tabular}{lllcl}
\hline LSA1437 & Isa1437 & N-acetylmuramoyl-L-alanine amidase precursor (cell wall hydrolase) (autolysin) & -0.7 \\
LSA1441 & bacA & Putative undecaprenol kinase (bacitracine resistance protein A) & 0.6 \\
LSA1613 & alr & Alanine racemase & -0.8 & -0.9 \\
LSA1616 & murF & UDP-N-acetylmuramoyl-tripeptide-D-alanyl-D-alanine ligase & -0.7 \\
\multicolumn{2}{l}{ Cell envelope and cellular processes } & -0.5 \\
LSA0162 & Isa0162 & Putative Bifunctional glycosyl transferase, family 8 & $\mathbf{- 1 . 2}$ \\
LSA1246 & Isa1246 & Putative glycosyl transferase, family 2 & $\mathbf{- 1 . 5}$ \\
LSA1558 & Isa1558 & Putative extracellular N-acetylmuramoyl-L-alanine amidase precursor (cell wall hydrolase/Lysosyme & -0.9
\end{tabular}

Cell motility and secretion

Protein secretion

LSA0948 IspA Signal peptidase II (lipoprotein signal peptidase) (prolipoprotein signal peptidase)

LSA1884 OXaA2

Membrane protein chaperone oxaA

Signal transduction

Signal transduction

LSA0561 SPPKN

Two-component system, sensor histidine kinase, (SppK fragment), degenerate

0.5

LSA0692 Isa0692

Putative serine/threonine protein kinase

0.5

LSA1384 Isa1384

Two-component system, response regulator

Post translational modifications, protein turnover, chaperones

\section{Protein folding}

LSA0050 Isa0050 Putative molecular chaperone, small heat shock protein, Hsp20 family

LSA0082 htrA Serine protease HtrA precursor, trypsin family

LSA0207 ClpL ATPase/chaperone ClpL, putative specificity factor for ClpP protease

LSA0358 groS Co-chaperonin GroES (10 kD chaperonin) (protein Cpn10)

LSA0359 groEL Chaperonin GroEL (60 kDa chaperonin) (protein Cpn60)

LSA0436 Isa0436 Putative peptidylprolyl isomerase (peptidylprolyl cis-trans isomerase) (PPlase)

LSA0984 hs/U ATP-dependent Hsl protease, ATP-binding subunit HsIU

LSA1465 ClpE

ATPase/chaperone ClpE, putative specificity factor for ClpP protease

LSA1618 htpX

Membrane metalloprotease, HtpX homolog

\section{Adaption to atypical conditions}

$\begin{array}{lll}\text { LSA0170 } & \text { Isa0170 } & \text { Putative general stress protein } \\ \text { LSA0247 } & \text { usp2 } & \text { Similar to universal stress protein, UspA family } \\ \text { LSA0264 } & \text { Isa0264 } & \text { Putative glycine/betaine/carnitine/choline transport protein } \\ \text { LSA0513 } & \text { Isa0513 } & \text { Putative stress-responsive transcriptional regulator } \\ \text { LSA0552 } & \text { Isa0552 } & \text { Organic hydroperoxide resistance protein } \\ \text { LSA0616 } & \text { Isa0616 } & \text { Putative glycine/betaine/carnitine/choline ABC transporter, ATP-binding subunit } \\ \text { LSA0617 } & \text { Isa0617 } & \text { Putative glycine/betaine/carnitine/choline ABC transporter, membrane-spanning subunit } \\ \text { LSA0618 } & \text { Isa0618 } & \text { Putative glycine/betaine/carnitine/choline ABC transporter, substrate-binding lipoprotein } \\ \text { LSA0619 } & \text { Isa0619 } & \text { Putative glycine/betaine/carnitine/choline ABC transporter, membrane-spanning subunit } \\ \text { LSA0642 } & \text { usp3 } & \text { Similar to universal stress protein, UspA } \\ \text { LSA0768 } & \text { csp1 } & \text { Similar to cold shock protein, CspA family } \\ \text { LSA0836 } & \text { usp6 } & \text { Similar to universal stress protein, UspA family } \\ \text { LSA0946 } & \text { csp4 } & \text { Similar to cold shock protein, CspA family } \\ \text { LSA1110 } & \text { Isa1110 } & \text { Putative NifU-homolog involved in Fe-S cluster assembly } \\ \text { LSA1111 } & \text { Isa1111 } & \text { Putative cysteine desulfurase (class-V aminotransferase, putative SufS protein homologue) } \\ \text { LSA1173 } & \text { usp4 } & \text { Similar to universal stress protein, UspA family } \\ \text { LSA1694 } & \text { Isa1694 } & \text { Putative glycine/betaine/carnitine ABC transporter, substrate binding lipoprotein precursor } \\ \text { LSA1695 } & \text { Isa1695 } & \text { Putative glycine/betaine/carnitine ABC transporter, membrane-spanning subunit }\end{array}$


Table 1 Genes with significant differential expression in three $L$. sakei strains grown on ribose compared with glucose, FDR adjusted $p$-value less than 0.01 and $\log _{2}$ of $>0.5$ or $<-0.5\left(\log _{2}\right.$ values $>1.0$ or $<-1.0$ are shown in bold) (Continued)

\begin{tabular}{|c|c|c|c|c|c|}
\hline LSA1696 & Isa1696 & Putative glycine/betaine/carnitine ABC transporter, ATP-binding subunit & -1.6 & & -0.9 \\
\hline LSA1870 & Isa1870 & Putative glycine betaine/carnitine/choline ABC transporter, ATP-binding subunit & -0.6 & & -0.6 \\
\hline \multicolumn{6}{|c|}{ Protein modification } \\
\hline LSA0865 & Isa0865 & Putative protein methionine sulfoxide reductase & & -0.6 & \\
\hline LSA0866 & msrA & Protein methionine sulfoxide reductase & & -0.7 & \\
\hline LSA0934 & Ip/A & Lipoate-protein ligase & 1.6 & 1.4 & 1.0 \\
\hline LSA0973 & pfIA & Pyruvate formate-lyase activating enzyme & 1.7 & & \\
\hline
\end{tabular}

\section{General function prediction only}

\section{Miscellaneous}

\begin{tabular}{|c|c|c|c|c|c|}
\hline LSA0030 & $15 a 0030$ & Putative aldo/keto reductase (oxidoreductase) & & -0.7 & -0.8 \\
\hline LSA0120 & $15 a 0120$ & Putative GTP-binding protein & -0.5 & & \\
\hline LSA0164 & $15 a 0164$ & Putative serine/tyrosine protein phosphatase & 0.2 & -1.1 & -1.2 \\
\hline$\underline{\text { LSA0165 }}$ & $15 a 0165$ & Putative oxidoreductase, short chain dehydrogenase/reductase family & & -0.9 & -1.2 \\
\hline LSA0218 & $\operatorname{trxA1}$ & Thioredoxin & & -0.9 & \\
\hline LSA0258 & $1 s a 0258$ & Putative iron-containing alcohol dehydrogenase & 1.6 & 0.5 & 1.6 \\
\hline LSA0260 & $15 a 0260$ & Putative aldo/keto reductase (oxidoreductase) & 1.9 & 1.2 & 1.7 \\
\hline LSA0312 & $15 a 0312$ & Putative NADH oxidase & -0.9 & & -1.0 \\
\hline LSA0324 & $15 a 0324$ & Putative hydrolase, haloacid dehalogenase family (N-terminal fragment), authentic frameshift & 1.9 & & \\
\hline LSA0325 & $15 a 0325$ & Putative hydrolase, haloacid dehalogenase family (C-terminal fragment), authentic frameshift & 1.8 & & \\
\hline LSA0350 & $15 a 0350$ & Putative N-acetyltransferase, GNAT family & -0.5 & & \\
\hline LSA0369 & $15 a 0369$ & Putative N-acetyltransferase, GNAT family & -0.5 & & -0.5 \\
\hline LSA0384 & $15 a 0384$ & Putative phosphoesterase, DHH family & -0.5 & & \\
\hline LSA0403 & $15 a 0403$ & Putative thioredoxin reductase & & 0.9 & \\
\hline LSA0447 & Isa0447 & Putative hydrolase, haloacid dehalogenase family & & & 0.6 \\
\hline LSA0475 & Isa0475 & Putative N-acetyltransferase, GNAT family & & -0.6 & \\
\hline LSA0520 & $\operatorname{tr} \times B 2$ & Thioredoxin reductase & -0.8 & & \\
\hline LSA0575 & $n p r$ & NADH peroxidase & 1.0 & U & \\
\hline LSA0802 & nox & NADH oxidase & 1.5 & & \\
\hline LSA0806 & $15 a 0806$ & Putative N-acetyltransferase, GNAT family & 0.6 & & \\
\hline LSA0831 & Isa0831 & Putative nitroreductase (oxidoreductase) & & 1.6 & \\
\hline LSA0896 & sodA & Iron/Manganese superoxide dismutase & 3.4 & 1.7 & 1.7 \\
\hline LSA0925 & $a d h$ & Putative zinc-containg alcohol dehydrogenase (oxidoreductase) & 0.5 & & \\
\hline LSA0971 & ppa & Inorganic pyrophosphatase (pyrophosphate phosphohydrolase) & 0.7 & & \\
\hline LSA0994 & $15 a 0994$ & Putative GTP-binding protein & & & 0.6 \\
\hline LSA1016 & engA & Putative GTP-binding protein & 0.6 & & 0.7 \\
\hline LSA1045 & obgE & Putative GTP-binding protein & 0.6 & & \\
\hline LSA1153 & Isa1153 & Hypothetical protein, CAAX protease family & 0.5 & & \\
\hline LSA1311 & Isa1311 & Hypothetical protein containing a possible heme/steroid binding domain & 0.7 & -0.6 & \\
\hline LSA1320 & Isa1320 & Putative NADPH-quinone oxidoreductase & & -0.8 & \\
\hline LSA1345 & Isa1345 & Putative hydrolase, haloacid dehalogenase family & 0.5 & & \\
\hline LSA1349 & Isa1349 & Putative N-acetyltransferase, GNAT family & & -0.5 & \\
\hline LSA1365 & Isa1365 & Hypothetical protein & & -0.5 & -0.7 \\
\hline LSA1368 & $15 a 1368$ & Hypothetical protein & 0.9 & & 0.6 \\
\hline LSA1371 & Isa1371 & Hypothetical membrane protein & 0.6 & & \\
\hline LSA1395 & Isa1395 & Putative zinc-containing alcohol dehydrogenase (oxidoreductase) & 0.9 & & \\
\hline LSA1427 & Isa1427 & Putative hydrolase, haloacid dehalogenase & 1.3 & & 0.6 \\
\hline LSA1472 & Isa1472 & Putative N-acetyl transferase, GNAT family & 0.6 & & \\
\hline LSA1535 & Isa1535 & Putative oxidoreductase & 0.5 & 1.1 & 0.7 \\
\hline LSA1553 & Isa1553 & Putative hydrolase, haloacid dehalogenase family & -0.6 & & \\
\hline
\end{tabular}


Table 1 Genes with significant differential expression in three $L$. sakei strains grown on ribose compared with glucose, FDR adjusted $p$-value less than 0.01 and $\log _{2}$ of $>0.5$ or $<-0.5\left(\log _{2}\right.$ values $>1.0$ or $<-1.0$ are shown in bold) (Continued)

\begin{tabular}{lllcl}
\hline LSA1559 & Isa1559 & Putative oxidoreductase & 0.6 & $\mathbf{1 . 1}$ \\
LSA1702 & Isa1702 & Putative zinc-containing alcohol dehydrogenase (oxidoreductase) & 0.7 \\
LSA1712 & Isa1712 & Putative nitroreductase (oxidoreductase) & $\mathbf{1 . 1}$ & -0.7 \\
LSA1832 & Isa1832 & Putative zinc-containing alcohol dehydrogenase (oxidoreductase) & -0.8 \\
LSA1835 & Isa1835 & Putative zinc-containing alcohol dehydrogenase (oxidoreductase) & $\mathbf{1 . 0}$ \\
LSA1867 & Isa1867 & Putative acetyltransferase, isoleucine patch superfamily & -0.7 & $-\mathbf{- 1 . 0}$ \\
LSA1871 & gshR & Glutathione reductase & -0.5 & -0.6 \\
\end{tabular}

\section{Unknown}

Proteins of unknown function that are similar to other proteins

\begin{tabular}{|c|c|c|}
\hline LSA0018 & Isa0018 & Hypothetical protein \\
\hline LSA0027 & 1sa0027 & Hypothetical protein \\
\hline LSA0028 & $15 a 0028$ & Hypothetical protein, DegV family \\
\hline LSA0044 & Isa0044 & Hypothetical protein \\
\hline LSA0061 & Isa0061 & Hypothetical extracellular protein precursor \\
\hline LSA0106 & Isa0106 & Hypothetical cell surface protein precursor \\
\hline LSA0160 & Isa0160 & Hypothetical protein \\
\hline LSA0166 & Isa0166 & Hypothetical Integral membrane protein \\
\hline A0190 & Isa0190 & Hypothetical integral membrane protein \\
\hline SA0191 & Isa0191 & Hypothetical integral membrane protein \\
\hline LSA0199 & Isa0199 & Hypothetical protein \\
\hline LSA0208 & 1sa0208 & Hypothetical integral membrane protein \\
\hline LSA0235 & Isa0235 & Hypothetical extracellular protein precursor \\
\hline LSA0236 & Isa0236 & Hypothetical extracellular peptide precursor \\
\hline LSA0244 & Isa0244 & Hypothetical integral membrane protein \\
\hline LSA0245 & Isa0245 & Hypothetical lipoprotein precursor \\
\hline LSA0249 & Isa0249 & Hypothetical protein \\
\hline LSA0263 & Isa0263 & Hypothetical integral membrane protein \\
\hline LSA0300 & Isa0300 & Hypothetical protein \\
\hline LSA0315 & Isa0315 & Hypothetical protein \\
\hline LSA0319 & Isa0319 & Hypothetical protein \\
\hline LSA0323 & Isa0323 & Hypothetical protein \\
\hline LSA0337 & Isa0337 & Hypothetical protein \\
\hline LSA0348 & Isa0348 & Hypothetical integral membrane protein \\
\hline LSA0352 & 1sa0352 & Hypothetical integral membrane protein \\
\hline LSA0354 & Isa0354 & Hypothetical integral membrane protein \\
\hline LSA0388 & $15 a 0388$ & Hypothetical protein \\
\hline LSA0389 & Isa0389 & Hypothetical protein \\
\hline LSA0390 & Isa0390 & Hypothetical protein \\
\hline LSA0409 & Isa0409 & Hypothetical integral membrane protein \\
\hline LSA0418 & Isa0418 & Hypothetical protein \\
\hline LSA0464 & Isa0464 & Hypothetical protein \\
\hline LSA0470 & Isa0470 & Hypothetical protein \\
\hline LSA0512 & 1sa0512 & Hypothetical protein \\
\hline LSA0515 & Isa0515 & Hypothetical integral membrane protein \\
\hline LSA0536 & Isa0536 & Hypothetical protein \\
\hline LSA0716 & Isa0716 & Hypothetical protein \\
\hline LSA0752 & Isa0752 & Hypothetical protein \\
\hline LSA0757 & Isa0757 & Hypothetical protein \\
\hline LSA0773 & Isa0773 & Hypothetical protein \\
\hline
\end{tabular}


Table 1 Genes with significant differential expression in three $L$. sakei strains grown on ribose compared with glucose, FDR adjusted p-value less than 0.01 and $\log _{2}$ of $>0.5$ or $<-0.5\left(\log _{2}\right.$ values $>1.0$ or $<-1.0$ are shown in bold) (Continued)

\begin{tabular}{|c|c|c|c|c|c|}
\hline LSA0784 & Isa0784 & Hypothetical protein & -2.6 & & \\
\hline LSA0786 & Isa0786 & Hypothetical protein & -2.0 & & \\
\hline LSA0787 & Isa0787 & Hypothetical protein & -1.7 & & \\
\hline LSA0790 & Isa0790 & Hypothetical protein, ATP utilizing enzyme PP-loop family & -2.5 & & \\
\hline LSA0827 & Isa0827 & Hypothetical lipoprotein precursor & 0.8 & & U \\
\hline LSA0828 & $15 a 0828$ & Hypothetical protein & 0.7 & & \\
\hline LSA0829 & $15 a 0829$ & Hypothetical integral membrane protein & & & 0.5 \\
\hline LSA0874 & Isa0874 & Hypothetical protein & 0.5 & & \\
\hline LSA0901 & Isa0901 & Hypothetical protein & & & 0.5 \\
\hline LSA0913 & Isa0913 & Hypothetical extracellular protein precursor & 0.5 & & 0.7 \\
\hline LSA0919 & Isa0919 & Hypothetical protein & & & 0.7 \\
\hline LSA0933 & Isa0933 & Hypothetical protein & 0.6 & & 0.6 \\
\hline LSA0961 & Isa0961 & Hypothetical protein, DegV family & & -0.5 & \\
\hline LSA0968 & Isa0968 & Hypothetical integral membrane protein & 0.7 & & \\
\hline LSA0977 & Isa0977 & Hypothetical integral membrane protein & 0.7 & & 0.8 \\
\hline LSA0987 & $1 s a 0987$ & Hypotehtical protein, GidA family (C-terminal fragment) & 0.5 & & \\
\hline LSA0996 & Isa0996 & Hypothetical protein & & & 0.5 \\
\hline LSA1003 & Isa1003 & Hypothetical protein & 2.0 & & 1.2 \\
\hline LSA1005 & Isa1005 & Hypothetical membrane protein & 0.9 & 0.6 & 0.7 \\
\hline LSA1008 & Isa1008 & Putative extracellular chitin-binding protein precursor & & 0.9 & 1.2 \\
\hline LSA1027 & Isa1027 & Hypothetical protein & & & 0.6 \\
\hline LSA1047 & Isa1047 & Hypothetical protein & 3.5 & 1.2 & 1.3 \\
\hline LSA1064 & Isa1064 & Hypothetical protein & 0.5 & & 0.7 \\
\hline LSA1075 & Isa1075 & Hypothetical protein & & & 0.5 \\
\hline LSA1078 & Isa1078 & Hypothetical protein & & & 0.6 \\
\hline LSA1081 & Isa1081 & Hypothetical protein & 1.0 & & 1.0 \\
\hline LSA1091 & Isa1091 & Hypothetical protein & & & 0.6 \\
\hline LSA1096 & Isa1096 & Hypothetical protein & 0.6 & & \\
\hline LSA1124 & Isal124 & Hypothetical protein & & -0.7 & \\
\hline LSA1154 & Isa1154 & Hypothetical protein & 0.6 & & 0.6 \\
\hline LSA1158 & Isa1158 & Hypothetical protein & 1.7 & 1.4 & \\
\hline LSA1189 & Isa1189 & Hypothetical integral membrane protein & -1.6 & & -1.1 \\
\hline LSA1282 & $1 s a 1282$ & Hypothetical protein & & -0.5 & \\
\hline LSA1296 & Isa1296 & Hypothetical integral membrane protein & & -1.2 & -0.8 \\
\hline LSA1342 & Isa1342 & Hypothetical protein & & -0.7 & \\
\hline LSA1346 & Isa1346 & Hypothetical protein & 0.8 & & \\
\hline LSA1350 & Isa1350 & Hypothetical protein & & -0.6 & -1.0 \\
\hline LSA1353 & Isa1353 & Hypothetical integral membrane protein & -0.9 & -0.5 & \\
\hline LSA1446 & Isa1446 & Hypothetical protein & -0.6 & -0.6 & -0.7 \\
\hline LSA1466 & Isa1466 & Hypothetical protein & 0.6 & & \\
\hline LSA1467 & Isa1467 & Hypothetical protein & & -0.6 & -1.1 \\
\hline LSA1524 & Isa1524 & Hypothetical protein & 0.7 & & \\
\hline LSA1540 & Isa1540 & Hypothetical extracellular protein precursor & 0.7 & & \\
\hline LSA1563 & Isa1563 & Hypothetical integral membrane protein & & -0.6 & -0.6 \\
\hline LSA1610 & Isa1610 & Hypothetical integral membrane protein & -0.7 & & -0.9 \\
\hline LSA1617 & Isa1617 & Hypothetical protein & & & -0.7 \\
\hline LSA1620 & Isa1620 & Hypothetical protein & & & -0.6 \\
\hline LSA1623 & Isa1623 & Hypothetical integral membrane protein & -0.5 & & -0.6 \\
\hline LSA1637 & Isa1637 & Hypothetical integral membrane protein, TerC family & -1.7 & -1.0 & -1.6 \\
\hline LSA1644 & Isa1644 & Hypothetical protein & 1.7 & & $\mathrm{D}$ \\
\hline
\end{tabular}


Table 1 Genes with significant differential expression in three $L$. sakei strains grown on ribose compared with glucose, FDR adjusted $p$-value less than 0.01 and $\log _{2}$ of $>0.5$ or $<-0.5\left(\log _{2}\right.$ values $>1.0$ or $<-1.0$ are shown in bold) (Continued)

\begin{tabular}{|c|c|c|c|c|c|}
\hline LSA1649 & Isa1649 & Hypothetical extracellular protein precursor & & & -0.5 \\
\hline LSA1659 & Isa1659 & Hypothetical protein & -0.5 & & \\
\hline LSA1662 & Isa1662 & Hypothetical protein & -1.0 & -0.6 & -0.7 \\
\hline LSA1663 & Isa1663 & Hypothetical protein & -0.8 & & \\
\hline LSA1678 & Isa1678 & Hypothetical protein & -0.6 & & \\
\hline LSA1680 & Isa1680 & Hypothetical protein & -0.6 & & \\
\hline LSA1716 & Isa1716 & Hypothetical protein & & -0.5 & \\
\hline LSA1822 & Isa1822 & Hypothetical protein & & & -0.5 \\
\hline LSA1828 & Isa1828 & Hypothetical integral membrane protein & 0.6 & 0.7 & \\
\hline LSA1850 & $1 s a 1850$ & Hypothetical protein & & -0.6 & \\
\hline LSA1876 & Isa1876 & Hypothetical integral membrane protein & & & -0.6 \\
\hline LSA1877 & Isa1877 & Hypothetical protein & & & -0.6 \\
\hline \multicolumn{6}{|c|}{ Proteins of unknown function only similar to other proteins from the same organism } \\
\hline LSA1159 & Isal159 & Hypothetical cell surface protein precursor & 2.0 & & 0.5 \\
\hline LSA1165 & Isa1165 & Hypothetical cell surface protein precursor & 1.8 & & \\
\hline LSA1700 & Isa1700 & Hypothetical protein & 2.1 & 0.8 & \\
\hline LSA1814 & Isa1814 & Hypothetical protein & & & -0.5 \\
\hline \multicolumn{6}{|c|}{ Proteins of unknown function. without similarity to other proteins } \\
\hline LSA0065 & Isa0065 & Hypothetical integral membrane protein & -0.5 & & \\
\hline LSA0093 & Isa0093 & Hypothetical integral membrane protein & -0.9 & & -1.2 \\
\hline LSA0121 & $15 a 0121$ & Hypothetical small peptide & -0.7 & -0.6 & -0.5 \\
\hline LSA0163 & Isa0163 & Hypothetical protein & & -1.1 & -1.3 \\
\hline LSA0167 & $15 a 0167$ & Hypothetical protein & & & -1.4 \\
\hline LSA0168 & $15 a 0168$ & Hypothetical protein & & & -1.4 \\
\hline LSA0188 & Isa0188 & Hypothetical small peptide & & & -0.8 \\
\hline LSA0256_a & Isa0256_a & Hypothetical protein & 2.3 & 1.0 & 2.2 \\
\hline LSA0257 & Isa0257 & Hypothetical protein & 1.4 & & \\
\hline LSA0281 & $15 a 0281$ & Hypothetical lipoprotein precursor & & -0.5 & -0.6 \\
\hline LSA0301 & Isa0301 & Hypothetical protein & & & 0.6 \\
\hline LSA0334 & Isa0334 & Hypothetical extracellular protein precursor & 1.1 & & \\
\hline LSA0339 & Isa0339 & Hypothetical protein & -0.5 & & \\
\hline LSA0378 & $15 a 0378$ & Hypothetical protein & -0.7 & & \\
\hline LSA0514 & $15 a 0514$ & Hypothetical small extracellular protein precursor & & -0.8 & \\
\hline LSA0534 & Isa0534 & Hypothetical cell surface protein precursor (with LPQTG sorting signal) & 1.0 & & D \\
\hline LSA0576 & Isa0576 & Hypothetical protein & 0.5 & $\mathrm{D}$ & \\
\hline LSA0641 & Isa0641 & Hypothetical extracellular peptide precursor & & -0.5 & \\
\hline LSA0647 & Isa0647 & Hypothetical extracellular protein precursor & 0.6 & & \\
\hline LSA0667 & Isa0667 & Hypothetical protein & 1.0 & & 0.9 \\
\hline LSA0753 & Isa0753 & Hypothetical integral membrane protein & & & 0.5 \\
\hline LSA0789 & Isa0789 & Hypothetical protein & -1.9 & & \\
\hline LSA0837 & Isa0837 & Hypothetical protein & 1.2 & 1.3 & 1.4 \\
\hline LSA0885 & Isa0885 & Hypothetical protein & 1.8 & & \\
\hline LSA0902 & Isa0902 & Hypothetical protein & 0.7 & D & \\
\hline LSA0945 & $15 a 0945$ & Hypothetical protein & & & 0.9 \\
\hline LSA1019 & Isa1019 & Hypothetical cell surface protein precursor & & & 0.8 \\
\hline LSA1035 & Isa1035 & Hypothetical small integral membrane protein & & & 0.6 \\
\hline LSA1086 & Isa1086 & hypothetical protein & 0.8 & & 0.5 \\
\hline LSA1104 & Isa1104 & Hypothetical protein & -0.5 & & \\
\hline LSA1155 & Isa1155 & Hypothetical integral membrane protein & 0.5 & & \\
\hline LSA1174 & Isa1174 & Hypothetical protein & 1.0 & & \\
\hline
\end{tabular}


Table 1 Genes with significant differential expression in three $L$. sakei strains grown on ribose compared with glucose, FDR adjusted p-value less than 0.01 and $\log _{2}$ of $>0.5$ or $<-0.5\left(\log _{2}\right.$ values $>1.0$ or $<-1.0$ are shown in bold) (Continued)

\begin{tabular}{|c|c|c|c|c|c|}
\hline LSA1176 & Isa1176 & Hypothetical protein & & -1.0 & $U$ \\
\hline LSA1319 & Isa1319 & Hypothetical small protein & & -0.8 & \\
\hline LSA1408 & Isa1408 & Hypothetical protein & & & 0.6 \\
\hline LSA1464 & Isa1464 & Hypothetical protein & -0.6 & & \\
\hline LSA1478 & Isa1478 & Hypothetical protein & -0.7 & -0.6 & -0.6 \\
\hline LSA 1480 & Isal480 & Hypothetical membrane protein & 0.5 & $\mathrm{D}$ & \\
\hline LSA1524 & Isa1524 & Hypothetical protein & 0.8 & & \\
\hline LSA1539 & Isa1539 & Hypothetical protein & 0.9 & & \\
\hline LSA1713 & Isa1713 & Hypothtical small peptide & & & -0.6 \\
\hline LSA1787 & Isa1787 & Hypothetical cell surface protein precursor & -0.5 & $U$ & \\
\hline LSA1820 & Isa1820 & Hypothetical cell surface protein precursor & & & -0.6 \\
\hline LSA1821 & Isa1821 & Hypothetical cell surface protein precursor & & -0.6 & \\
\hline LSA1845 & Isa1845 & Hypothetical small protein & & 0.8 & \\
\hline LSA1848 & Isa1848 & Hypothetical protein & & & -0.5 \\
\hline LSA1851 & Isa1851 & Hypothetical extracellular small protein & -0.6 & & -0.7 \\
\hline LSA1883 & Isa1883 & Hypothetical small protein & 1.2 & & 1.5 \\
\hline \multicolumn{6}{|c|}{ Bacteriocin associated genes } \\
\hline SKP0001 & spplP & Bacteriocin sakacin $P$ inducing peptide & D & 0.5 & D \\
\hline SKP0006 & sppt & Sakacin P ABC transporter & D & 0.6 & D \\
\hline SKP0007 & sppE & Sakacin P accesory transport protein & D & 0.6 & $\mathrm{D}$ \\
\hline
\end{tabular}

The microarray used has been described previously [32]. Asterix $\left.{ }^{*}\right)$ relates the gene to Table 2. D and $U$ refer to genes classified as 'divergent' and 'uncertain', respectively, by CGH analysis [32]. Genes encoding proteins with a change in expression according to McLeod et al. [19], are underlined.

protein expression of glyceraldehyde-3-phosphate dehydrogenase, phosphoglycerate kinase and enolase previously seen in LS 25 [19]. The latter three enzymes are encoded from the central glycolytic operon (cggR-gappgk-tpi-eno) together with triose-phosphate isomerase and the putative central glycolytic genes regulator (CggR) [46]. Besides the $\operatorname{cggR}$ gene being down-regulated in MF1053 and LS 25, no change in gene expression was seen of these central glycolytic genes. Thus at the transcription level it is not obvious that the LS 25 strain

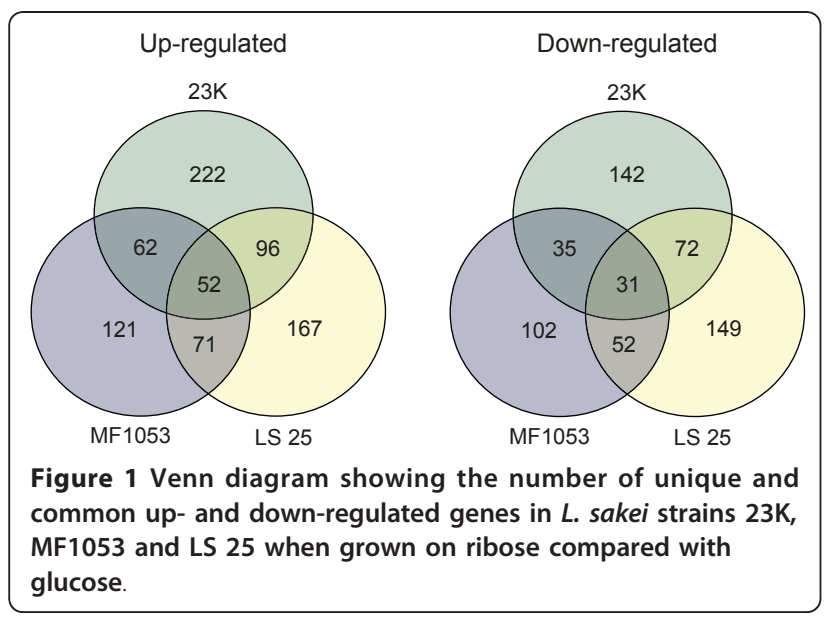

down-regulate the glycolytic pathway more efficiently than the other strains, as previously suggested [19].

Interestingly, all the strains showed an induction (1.42.3) of $m g s A$ encoding methylglyoxal synthase, which catalyzes the conversion of dihydroxyacetone-phosphate to methylglyoxal (Figure 2). The presence of this gene is uncommon among LAB and so far a unique feature among the sequenced lactobacilli. The methylglyoxal pathway represents an energetically unfavourable bypass to the glycolysis. In E. coli, this bypass occurs as a response to phosphate starvation or uncontrolled carbohydrate metabolism, and enhanced ribose uptake was shown to lead to the accumulation of methylglyoxal $[47,48]$. As suggested by Chaillou et al. [7], such flexibility in the glycolytic process in L. sakei may reflect the requirement to deal with glucose starvation or to modulate carbon flux during cometabolism of alternative carbon sources. Breakdown of methylglyoxal is important as it is toxic to the cells [49]. An induction of the $l s a 1158$ gene contiguous with $m g s A$ was seen for $23 \mathrm{~K}$ and MF1053. This gene encodes a hypothetical protein, also suggested as a putative oxidoreductase, which may reduce methylglyoxal to lactaldehyde [7]. However, no induction of the $a d h E$ (lsa0379) gene encoding an iron-containing aldehyde dehydrogenase suggested to further reduce lactaldehyde to L-lactate [7] was seen. By CGH [32]lsa1158 and adhE were present in all 


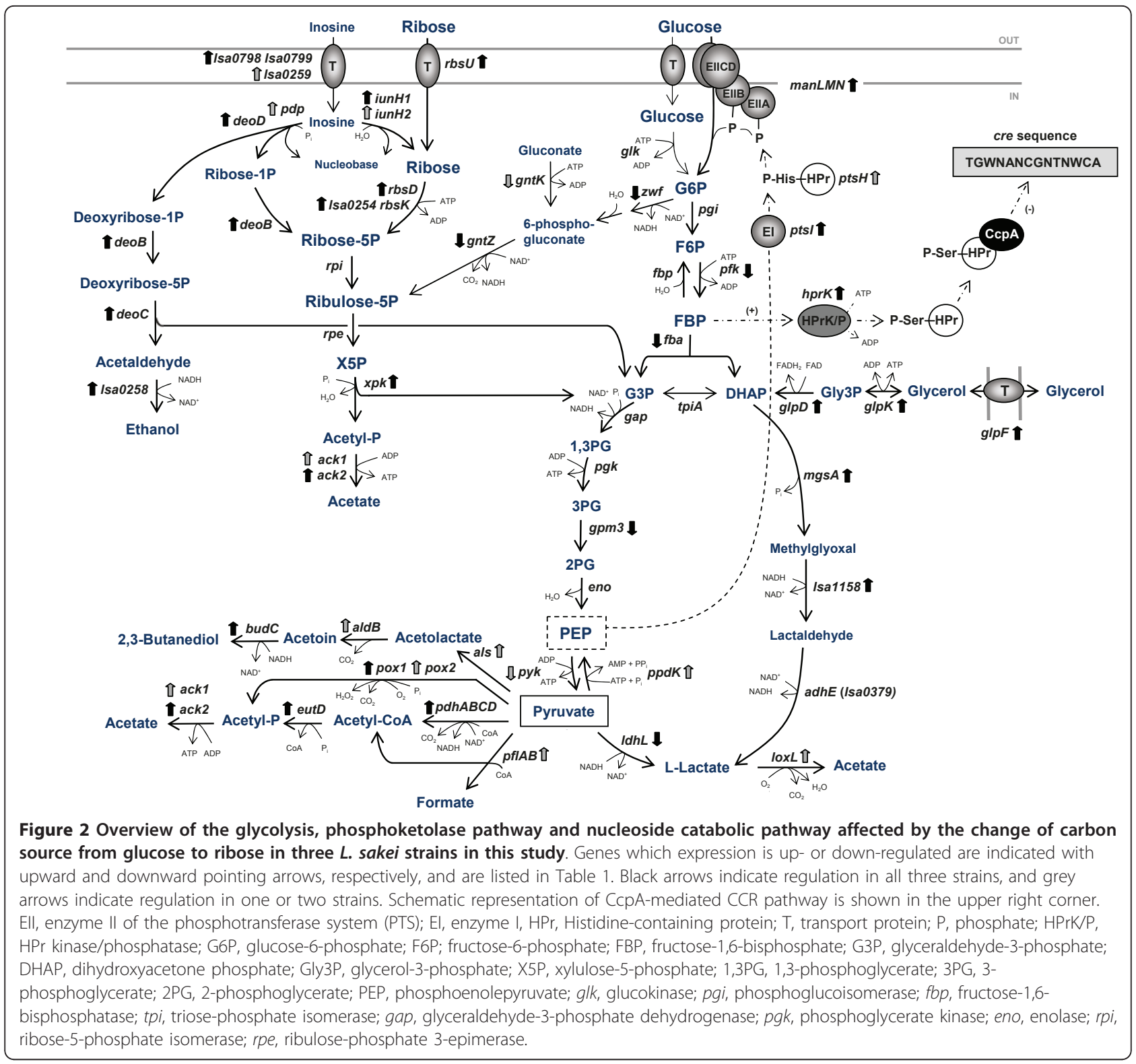

the $L$. sakei strains investigated, whereas $m g s A$ was lacking in some strains, indicating that the MgsA function is not vital.

\section{Pyruvate metabolism}

Pyruvate is important in both glycolysis and PKP. It can be converted into lactate by the NAD-dependent L-lactate dehydrogenase, which regenerates $\mathrm{NAD}^{+}$and maintains the redox balance. This enzyme is encoded by the $l d h L$ gene which was down-regulated (0.7-1.4) in all three strains, in accordance with previous findings [50], and the down-regulation was strongest for the LS 25 strain. At the protein level, only LS 25 showed a lower expression of this enzyme during growth on ribose [19].
Genes responsible for alternative fates of pyruvate (Figure 2) were highly induced in all the strains, however with some interesting strain variation (Table 1). The shift in pyruvate metabolism can benefit the bacteria by generating ATP, or by gaining $\mathrm{NAD}^{+}$for maintaining the redox balance and may lead to various end products in addition to lactate [51].

In all the strains, a strongly up-regulated (2.1-3.0) pox1 gene was observed, and in $23 \mathrm{~K}$ an up-regulated pox2 (0.7), encoding pyruvate oxidases which under aerobic conditions convert pyruvate to acetyl-phosphate with hydrogen peroxide $\left(\mathrm{H}_{2} \mathrm{O}_{2}\right)$ and $\mathrm{CO}_{2}$ as side products. Accumulation of peroxide ultimately leads to aerobic growth arrest [52]. $\mathrm{H}_{2} \mathrm{O}_{2}$ belongs to a group of compounds known as reactive 
oxygen species and reacts readily with metal ions to yield hydroxyl radicals that damage DNA, proteins and membranes [53]. Remarkable differences in redox activities exist among Lactobacillus species and L. sakei is among those extensively well equipped to cope with changing oxygen conditions, as well as dealing effectively with toxic oxygen byproducts [7]. 23K up-regulated npr (1.0) encoding $\mathrm{NADH}$ peroxidase which decomposes low concentrations of $\mathrm{H}_{2} \mathrm{O}_{2}$ to $\mathrm{H}_{2} \mathrm{O}$ and $\mathrm{O}_{2}$, and all the strains upregulated the $\operatorname{sod} A$ gene (1.7-3.4) encoding a superoxide dismutase which produces hydrogen peroxide from superoxide $\left(\mathrm{O}_{2}{ }^{-}\right)$. Various oxidoreductases showed an up-regulation in all the strains (Table 1), indicating the need for the bacterium to maintain its redox balance.

The $p d h A B C D$ gene cluster encoding components of the pyruvate dehydrogenase enzyme complex (PDC) which transforms pyruvate into acetyl-CoA and $\mathrm{CO}_{2}$ were among the strongly up-regulated (2.1-3.7) genes. The eutD gene encoding a phosphate acetyltransferase which further forms acetyl-phosphate from acetyl-CoA was also induced (1.0-2.0). Pyruvate can be transformed to acetolactate by acetolactate synthase and further to acetoin by acetolactate decarboxylase, before 2,3-butanediol may be formed by an acetoin recuctase (Figure 2). While the budC gene encoding the acetoin reductase showed a strong up-regulation in all three strains, the als-aldB operon was only strongly up-regulated in LS 25 (1.9). Pyruvate formate lyase produces acetyl-CoA and formate from pyruvate. Only in $23 \mathrm{~K}$, the pflAB genes encoding formate $\mathrm{C}$-acetyltransferase and its activating enzyme involved in formate formation were strongly upregulated (4.0 and 1.7, respectively). This strain was the only one to strongly induce L-lactate oxidase encoding genes which are responsible for conversion of lactate to acetate when oxygen is present (Table 1 ). In $23 \mathrm{~K}$ and LS 25 , the $p p d K$ gene coding for the pyruvate phosphate dikinase involved in regenerating PEP, was induced, as was also lsa0444 encoding a putative malate dehydrogenase that catalyzes the conversion of malate into oxaloacetate using $\mathrm{NAD}^{+}$and vice versa (Table 1 ).

During growth on ribose, L. sakei was shown to require thiamine (vitamine B1) [15]. The E1 component subunit $\alpha$ of the PDC, as well as Pox and Xpk, require thiamine pyrophosphate, the active form of thiamine, as a coenzyme [54]. This could explain the induction of the thiMDE operon and $l s a 0055$ in LS 25, as well as $l s a 0980$ in $23 \mathrm{~K}$, encoding enzymes involved in thiamine uptake and biosynthesis (Table 1). The up-regulation of $l s a 1664$ (1.1-1.6) encoding a putative dihydrofolate reductase involved in biosynthesis of riboflavin (vitamin B2) in all the strains could indicate a requirement for flavin nucleotides as enzyme cofactors. Riboflavin is the precursor for flavin mononucleotide (FMN) and flavin adenine dinucleotide (FAD) redox cofactors in flavoproteins, and the
E3 component of PDC as well as glycerol-3-phosphate dehydrogenase encoded from the up-regulated $g l p D$, are among enzymes requiring FAD. Another cofactor which seems to be important during growth on ribose is lipoate, essential of the E2 component of the PDC. An up-regulation of $l p l A(1.0$ - 1.6) encoding lipoate-protein ligase, which facilitates attachment of the lipoyl moiety to metabolic enzyme complexes, was seen in all the strains, allowing the bacterium to scavenge extracellular lipoate $[55,56]$.

\section{Nucleoside catabolism}

The L. sakei genome contains a multiplicity of catabolic genes involved in exogenous nucleoside salvage pathways, and the bacterium has been shown to catabolize inosine and adenosine for energy [7]. Three iunH genes are present in the $23 \mathrm{~K}$ genome, which encode inosineuridine preferring nucleoside hydrolases responsible for conversion of inosine to ribose and purine base. The iunH1 gene was up-regulated in all the strains when grown on ribose (1.8-2.6), as was also the iunH2 gene in 23K (1.2). The deoC gene encodes a deoxyribose-phosphate aldolase, and is located in an operon structure preceding the genes $d e o B$, deoD, lsa0798, lsa0799, deoR and $p d p$ which encode phosphopentomutase, purine nucleoside phosphorylase, pyrimidine-specific nucleoside symporter, a putative purine transport protein, the deoxyribonucleoside synthesis operon transcriptional regulator (DeoR), and a pyrimidine-nucleoside phosphorylase, respectively. The complete operon was induced in all the strains, except for $p d p$ only induced in $23 \mathrm{~K}$ (Table $1)$. The phosphorylases catalyze cleavage of ribonucleosides and deoxyribonucleosides to the free base pluss ribose-1-phosphate or deoxyribose-1-phosphate. The bases are further utilized in nucleotide synthesis or as nitrogen sources. The pentomutase converts ribose-1phosphate or deoxyribose-1-phosphate to ribose-5-phosphate or deoxyribose-5-phosphate, respectively, which can be cleaved by the aldolase to glyceraldehyde-3-phosphate and acetaldehyde. Glyceraldehyde-3-phosphate enters the glycolysis, while a putative iron containing alcohol dehydrogenase, encoded by $l s a 0258$ up-regulated in all the strains (0.5-1.6), could further reduce acetaldehyde to ethanol (Figure 2). The obvious induced nucleoside catabolism at the level of gene expression was not seen by proteomic analysis [19].

\section{Genes involved in glycerol/glycerolipid/fatty acid metabolism}

During growth on ribose, a strong induction of the $g l p K D F$ operon encoding glycerol kinase (GlpK), glycerol-3-phosphate dehydrogenase (GlpD), and glycerol uptake facilitator protein was observed (Table 1), which is in correlation with the over-expression of GlpD and GlpK seen by 
proteomic analysis [19]. GlpD is $\mathrm{FADH}_{2}$ linked and converts glycerol-3-phosphate to dihydroxyacetone-phosphate. An over-expression of GlpD was also reported when L. sakei was exposed to low temperature [57]. A $g l p D$ mutant showed enhanced survival at low temperature, and it was suggested that this was a result of the glycerol metabolism being redirected into phosphatidic acid synthesis which leads to membrane phospholipid biosynthesis [57]. Nevertheless, a down-regulation was observed of the lsa1493 gene (0.6-0.9) encoding a putative diacylglycerol kinase involved in the synthesis of phosphatidic acid, and of $c f a$ (1.3-1.4) encoding cyclopropane-fatty-acyl-phospholipid synthase directly linked to modifications in the bacterial membrane fatty acid composition that reduce membrane fluidity and helps cells adapt to their environment [58]. Interestingly, LS 25 upregulated several genes (LSA0812-0823), including $a c c D$ and $a c c A$ encoding the $\alpha$ - and $\beta$-subunits of the multi-subunit acetyl-CoA carboxylase (Table 1). This is a biotin-dependent enzyme that catalyzes the irreversible carboxylation of acetyl-CoA to produce malonyl-CoA, an essential intermediate in fatty acid biosynthesis. In $B$. subtilis, the malonyl-CoA relieves repression of the $f a b$ genes [59]. We observed that also $a c p P, f a b Z 1, f a b H, f a b D$ and fabI (Table 1) encoding enzymes involved in fatty acid biosynthesis were induced in LS 25. The altered flux to malonyl-CoA may be a result of the decreased glycolytic rate. MF1053, on the other hand, showed a down-regulation of several genes in the same gene cluster. A higher level of acetate is produced when the bacterium utilizes ribose, and acetate lowers the $\mathrm{pH}$ and has a higher antimicrobial effect than lactate. Changes in the phospholipid composition could be a response to changes in intracellular $\mathrm{pH}$. Protons need to be expelled at a higher rate when the $\mathrm{pH}$ drops. The LS 25 strain which showed faster growth rates than the other strains [9], was the only strain to up-regulate the $\mathrm{F}_{0} \mathrm{~F}_{1}$ ATP synthase (Table 1), which at the expense of ATP expels protons during low $\mathrm{pH}$.

\section{Regulation mechanisms}

Little is known about the regulation of catabolic pathways in $L$. sakei. Starting from ribose uptake, the rbs operon may be both relieved from repression and ribose induced. Presumably, a dual regulation of this operon by two opposite mechanisms, substrate induction by ribose and CCR by glucose may occur in $L$. sakei. The $\operatorname{cp} A$ gene was not regulated, consistent with this gene commonly showing constitutive expression in lactobacilli $[42,60]$. The local repressor RbsR is homologous with CcpA, both belonging to the same LacI/GalR family of transcriptional regulators. RbsR was proposed to bind a cre-like consensus sequence located close to a putative CcpA cre site, both preceding rbsU [28]. RbsR in the Gram-positive soil bacterium Corynebacterium glutamicum was shown to bind a cre-like sequence, and using microarrays, the transcription of no other genes but the rbs operon was affected positively in an $r b s R$ deletion mutant. It was concluded that RbsR influences the expression of only the rbs operon [61]. Similarily, in the $L$. sakei sequence, no other candidate members of RbsR regulation could be found [28]. However, experiments are needed to confirm RbsR binding in

L. sakei. In Bacillus subtilis, RbsR represent a novel interaction partner of P-Ser-HPr in a similar fashion to CcpA [62]. The P-Ser-HPr interaction is possible also in L. sakei as the bacterium exhibits HPr-kinase/phosphatase activity.

A putative cre site is present in the promoter of $l s a 0254$ encoding the second ribokinase (Table 2), and this gene is preceeded by the opposite oriented gene $l s a 0253$ encoding a transcriptional regulator with a sugar binding domain which belongs to the GntR family. This family of transcriptional regulators, as well as the LacI family which RbsR and CcpA belong to, are among the families to which regulators involved in carbohydrate uptake or metabolism usually belong [63]. The GntR-type regulator could possibly be involved in regulating the expression of the second ribokinase, or of the inosine-uridine preferring nucleoside hydrolase encoding $i u n H 1$ gene which is located further upstream of lsa0254. C. glutamicum possesses an operon encoding a ribokinase, a uridine transporter, and a uridine-preferring nucleoside hydrolase which is co-controlled by a local repressor together with the $\mathrm{RbsR}$ repressor of the rbs operon $[60,61,64]$. It is possible that such co-control could exist also in L. sakei. Ribose as well as nucleosides are products of the degradation of organic materials such as DNA, RNA and ATP. The simultaneous expression of the $r b s$ and deo operons as well as the other genes involved in ribose and nucleoside catabolism (Figure 2) allows the bacterium to access the different substrates simultaneously and use both ribose as well as nucleosides as carbon and energy source. DeoR shows $51 \%$ identity to the B. subtilis DeoR repressor protein $[65,66]$. Genes encoding deoxyribosephosphate aldolase, nucleoside uptake protein and pyrimidine nucleoside phosphorylase in $B$. subtilis are organized in a $d r a-n u p C-p d p$ operon followed by $d e o R$, and ribose was shown to release DeoR from DNA binding and thus repression of the operon genes are alleviated [65-67]. The B. subtilis pentomutase and purine-nucleoside phosphorylase are encoded from a drm-pupG operon which is not negatively regulated by DeoR, though both operons are subject to CcpA mediated CCR $[65,66,68]$. As a cre site is found preceding the L. sakei deoC (Table 2), the operon could be regulated by CcpA as well. It is interesting that $d e o R$ is the only strongly induced transcriptional regulator gene in all three strains, and the encoded regulator has sigma $(\sigma)$ factor activity. We can only speculate whether it could function as 
Table 2 Putative cre sites present in the promoter region of some $L$. sakei genes up-regulated in the present study

\begin{tabular}{|c|c|c|c|c|c|}
\hline Gene locus & Gene & cre site sequence $^{a}$ & Position $^{b}$ & Co-transcribed genes/operon ${ }^{c}$ & Gene locus \\
\hline LSA0123 & Isa0123 & TGAAAGCGTTACAA & -93 & & \\
\hline LSA0185 & galp & GAACATCGTTATCA & -46 & & \\
\hline LSA0200 & $\mathrm{rbs} U$ & GTAAACCGTITCA & -113 & rbsUDK & LSA0200-0202 \\
\hline LSA0254 & Isa0254 & TGTAAGCGTTITAT & -56 & Isa0254-Isa0255-Isa0256_a & LSA0254-0256_a \\
\hline LSA0289 & xpk & 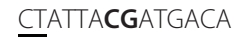 & -8 & & \\
\hline LSA0292 & budC & TGTAACCGTITAAA & -51 & & \\
\hline LSA0353 & Isa0353 & 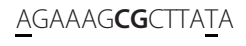 & -102 & & \\
\hline LSA0370 & $\operatorname{arc} A$ & TGAAAGCGATTACC & -58 & $\operatorname{arc} A-\operatorname{arc} B^{e}-\operatorname{arc} C-\operatorname{arct}^{e}-\operatorname{arc}^{e}$ & LSA0370-0374 \\
\hline LSA0449 & manL & TGTTAGCGTITIIA & -56 & manL-manM-manN & LSA0449-0451 \\
\hline LSA0533 & iunH2 & 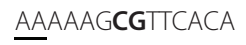 & -35 & & \\
\hline LSA0572 & $t d c B$ & TGAAAACGTTCTAAA & -134 & & \\
\hline LSA0608 & Glo AN & TGTAACCGTITA_A & -100 & gloAN-gloAC & LSA0608-0609 \\
\hline LSA0649 & glpK & $\underline{\text { AGGAAACGTTTCC }}$ & -42 & $g \mid p K-g l p D-g l p F$ & LSA0649-0651 \\
\hline LSA0664 & $10 \times L 1$ & $\underline{A G A A A G C G A G T A C A}$ & -82 & IOXL1N-IOXLI-IOXL1C & LSA0664-0666 \\
\hline LSA0764 & galk & TGAAAGCGATTAAT & -30 & galK-galE1-galT-galM & LSA0764-0767 \\
\hline LSA0795 & deoC & TGAAAGCGTTAACA & -33 & deoC-deoB-deoD-Isa0798-Isa0799-deoR-pdp & LSA0795-0801 \\
\hline LSA0974 & $p f l B$ & TACGAACGCTTACA & -147 & $p f I B-p f I A$ & LSA0974-0973 \\
\hline LSA1048 & fruR $R^{e}$ & TGTAAACGATGACA & -39 & fruR ${ }^{e}$-fruKe-fruA & LSA1048-1050 \\
\hline LSA1141 & ppdK & 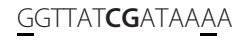 & -29 & & \\
\hline LSA1146 & manA & 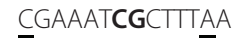 & -98 & & \\
\hline LSA1188 & pox1 & TGTAATCGATTTCA & -88 & & \\
\hline LSA1204 & Isa1204 & TGTAATCGTITIII & -127 & & \\
\hline LSA1343 & eutD & 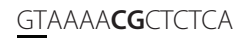 & -94 & & \\
\hline LSA1399 & $10 \times L 2$ & TGTAAACGATTTCA & -42 & & \\
\hline LSA1457 & Isa1457 & TGATAACGCTTACA & -85 & & \\
\hline LSA $1463^{d}$ & ptsH & TGAAAGCGGTATAG & -161 & ptsHl & LSA1463-1462 \\
\hline LSA1641 & nanE & TGTAAGCGGTTAAT & -85 & nanE-nanA & LSA1641-1640 \\
\hline LSA1643 & Isa1643 & TGATAACGCTTACA & -31 & & \\
\hline LSA1651 & Isa1651 & 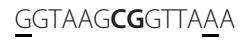 & -148 & & \\
\hline LSA1711 & lacL & TGAAACCGTITAAA & -36 & lacL-lacM & LSA1711-1710 \\
\hline LSA1792 & $s c r A$ & TGTAAACGGTTGTA & -78 & scrA-dexB-scrk & LSA1792-1790 \\
\hline LSA1830 & pox2 & TIGTAACGCTTACA & -70 & & \\
\hline
\end{tabular}

The identification is based on the genome sequence of $L$. sakei strain $23 \mathrm{~K}$, and the consensus sequence TGWNANCG NTNWCA (W $=A / T$, $N=A / T / G / C$ ), confirmed in Gram-positive bacteria [39] was used in the search, allowing up to two mismatches (underlined) in the conserved positions except for the two center positions, highlighted in boldface.

${ }^{a}$ mismatch to consensus sequence is underlined

b position of cre in relation to the start codon

c suggested co-transcribed genes or genes organized in an operon

${ }^{d}$ cre in preceding gene encoding hypothetical protein

e gene not regulated in this study

activator of transcription on some of the regulated genes in this study.

Expression of the Xpk encoding gene of Lactobacillus pentosus was reported to be induced by sugars fermented through the PKP and repressed by glucose mediated by CcpA [69]. Indeed, the cre site overlapping ATG start codon of L. sakei xpk (Table 2) indicates relief of CcpA-mediated CCR during growth on ribose. Also for several genes involved in alternative fates of pyruvate, putative cre sites were present (Table 2).

Several genes and operons involved in transport and metabolism of various carbohydrates such as mannose, galactose, fructose, lactose, cellobiose, N-acetylglucosamine, including putative sugar kinases and PTSs, were induced during growth on ribose (Table 1), and as shown in Table 2, putative cre sites are located in the promoter region of many of these up-regulated genes and operons. 23K showed an up-regulation of genes involved in the arginine deiminase pathway, and $23 \mathrm{~K}$ and LS 25 showed an up-regulated threonine deaminase (Table 1). The $\operatorname{arc} A$ and $t d c B$ both have putative $c r e$ sites in their promoter regions (Table 2). Thus ribose seems to induce a global regulation of carbon metabolism in L. sakei. 
A putative cre site precedes the glp operon (Table 2), suggesting regulation mediated by CcpA. However, regulation of the L. sakei GlpK may also occur by an inducer exclusion-based CcpA-independent CCR mechanism as described in enterococci and B. subtilis [70,71], and as previously suggested by Stentz et al. [15]. By this mechanism, glycerol metabolism is regulated by PEPdependent, EI- and HPr-catalyzed phosphorylation of GlpK in response to the presence or absence of a PTS substrate. In the absence of a PTS sugar, GlpK is phosphorylated by P-His-HPr at a conserved histidyl residue, forming the active P-GlpK form, whereas during growth on a PTS sugar, phosphoryl transfer flux through the PTS is high, concentration of P-His-HPr is low, and GlpK is present in a less active dephospho form $[20,70,71]$. This conserved histidyl residue (His232) is present in L. sakei GlpK [20], and Stentz et al. [15] reported that whereas $L$. sakei can grow poorly on glycerol, this growth was abolished in ptsI mutants.

\section{Mannose-PTS}

As mentioned in the introduction, the PTS plays a central role, in both the uptake of a number of carbohydrates and regulatory mechanisms [20-22]. Encoding the general components, $p t s H$ showed an up-regulation in MF1053 and LS 25 (1.2 and 0.9, respectively), while all the strains up-regulated $p t s I(0.8-1.7)$. The manLMN operon encoding the EII ${ }^{\text {man }}$ complex was surprisingly strongly up-regulated during growth on ribose in all the strains (Table 1). By proteomic analysis, no regulation of the PTS enzymes was seen [19]. The expression of $\mathrm{HPr}$ and EI in L. sakei during growth on glucose or ribose was previously suggested to be constitutive [14], and in other lactobacilli, the EII ${ }^{\text {man }}$ complex was reported to be consistently highly expressed, regardless of carbohydrate source [72-74]. Notably, PEP-dependent phosphorylation of PTS sugars has been detected in ribose-grown cells, indicating that the EII ${ }^{\text {man }}$ complex is active, and since no transport and phosphorylation via EII ${ }^{\text {man }}$ occurs, the complex is phosphorylated, while it is unphosphorylated in the presence of the substrates of the EII ${ }^{\text {man }}$ complex $[8,73]$. The stimulating effect exerted by small amounts of glucose on ribose uptake in L. sakei, which has also been reported in other lactobacilli $[74,75]$, was suggested to be caused by dephosphorylation of the PTS proteins in the presence of glucose, as a ptsI mutant lacking EI, as well as P-His-HPr, was shown to enhance ribose uptake $[15,16,76]$. Stentz et al. [15] observed that a L. sakei mutant (strain RV52) resistant to 2 deoxy-D-glucose, a glucose toxic analog transported by EII ${ }^{\text {man }}$, and thus assumed to be affected in the EII ${ }^{\text {man }}$, did not show the same enhanced uptake [15]. It was concluded that EII ${ }^{\text {man }}$ is not involved in the PTS-mediated regulation of ribose metabolism in L. sakei. The mutation was though not reported verified by sequencing [15], and other mutations could be responsible for the observed phenotype. The $L$. sakei EIIAB $^{\text {man }}$ EIIC $^{\text {man }}$ and EIID ${ }^{\text {man }}$ show 72, 81, and $82 \%$ identity, respectively, with the same enzymes in $L$. casei, in which mutations rendering the EII ${ }^{\text {man }}$ complex inactive were shown to derepress $r b s$ genes, resulting in a loss of the preferential use of glucose over ribose [75]. Furthermore, in L. pentosus, EII ${ }^{\text {man }}$ was shown to provide a strong signal to the CcpA-dependent repression pathway [73]. The hprK gene encoding HPrK/P which controls the phosphorylation state of HPr was strongly upregulated (1.2-2.0) in all three strains. HPrK/P dephosphorylates $\mathrm{P}-\mathrm{Ser}-\mathrm{HPr}$ when the concentration of glycolytic intermediates drop, which is likely the situation during growth on ribose [20,22,24].

Numerous genes encoding hypothetical proteins with unknown function were also found to be differentially expressed (Table 1), as well as several other genes belonging to various functional categories. For most of these, their direct connection with ribose metabolism is unknown, and is likely an indirect effect.

\section{Conclusions}

The ability to ferment meat and fish is related to the capacity of the bacterium to rapidly take up the available carbohydrates and other components for growth. The importance of this process, especially to the meat industry, stimulates research aimed at understanding the mechanisms for transport and metabolism of these compounds, with the ultimate goal to be able to select improved strains. Genome-wide transcriptome analyses with DNA microarrays efficiently allowed the identification of genes differentially expressed between growth on the two carbohydrates which $L$. sakei can utilize from these substrates. Moreover, microarrays were a powerful tool to increase the understanding of the bacterium's primary metabolism and revealed a global regulatory mechanism. In summary, the ribose uptake and catabolic machinery is highly regulated at the transcription level, and it is closely linked with catabolism of nucleosides. A global regulation mechanism seems to permit a fine tuning of the expression of enzymes that control efficient exploitation of available carbon sources.

\section{Additional material}

Additional file 1: Table S3. Primer and probe sets used for qRT-PCR Presents the primer and probe sets used for validation of microarray data by qRT-PCR analysis. Table S4. Comparison of microarray data with qRT-PCR results of $L$. sakei strain LS 25 grown on ribose compared with glucose. Presents gene regulation values $\left(\log _{2}\right)$ from the qRT-PCR analysis in comparison with microarray data. 


\section{Abbreviations}

PKP: phosphoketolase pathway; PEP: phosphoenolpyruvate; PTS: PEPdependent carbohydrate phosphotransferase system; CCR: carbon catabolite repression; cre: catabolite responsive element; RbsK: ribokinase; RbsD: DRibose pyranase; Xpk: xylulose-5-phosphate phosphoketolase; Ack: Acetate kinase, Pfk: 6-phosphofructokinase; Pyk: pyruvate kinase; PDC: pyruvate dehydrogenase complex; GlpD: glycerol-3-phosphate dehydrogenase; GlpK: glycerol kinase; Ell: enzyme Il; El: enzyme I; HPr: histidine protein; HPrK/P: HPr kinase/phosphatase; DeoR: deoxyribonucleoside synthesis operon transcriptional regulator.

\section{Acknowledgements and funding}

This work was financially supported by Grant 159058/110 from the Norwegian Research Council. The authors would like to thank Monique Zagorec for helpful suggestions and critically reading the manuscript. We also thank Margrete Solheim, Mari Christine Brekke, and Signe Marie Drømtorp for their assistance during the experiments, and Hallgeir Bergum, the Norwegian Microarray Consortium (NMC), for printing the microarray slides.

\section{Author details}

'Nofima Mat AS, Norwegian Institute of Food, Fisheries and Aquaculture Research, Osloveien 1, Ås, NO-1430, Norway. ${ }^{2}$ Department of Chemistry, Biotechnology and Food Science, Norwegian University of Life Sciences, P.O. Box 5003, Ås, NO-1432, Norway.

\section{Authors' contributions}

AM participated in the study design, conducted the experimental work, analyzed and interpreted data, and wrote the manuscript. LS conducted the statistical analysis. KN and LA conceived the study, participated in the study design process and reviewed the manuscript. All authors read and approved the final manuscript.

\section{Competing interests}

The authors declare that they have no competing interests.

Received: 9 February 2011 Accepted: 24 June 2011

Published: 24 June 2011

\section{References}

1. Hammes WP, Bantleon A, Min S: Lactic acid bacteria in meat fermentation. FEMS Microbiol Rev 1990, 87:165-174.

2. Hammes WP, Hertel C: New developments in meat starter cultures. Meat Science 1998, 49:125-138.

3. Bredholt S, Nesbakken T, Holck A: Protective cultures inhibit growth of Listeria monocytogenes and Escherichia coli 0157:H7 in cooked, sliced, vacuum- and gas-packaged meat. Int J Food Microbiol 1999, 53:43-52.

4. Bredholt S, Nesbakken T, Holck A: Industrial application of an antilisterial strain of Lactobacillus sakei as a protective culture and its effect on the sensory acceptability of cooked, sliced, vacuum-packaged meats. Int J Food Microbiol 2001, 66:191-196.

5. Katikou P, Georgantelis D, Paleologos EK, Ambrosiadis I, Kontominas MG: Relation of biogenic amines' formation with microbiological and sensory attributes in Lactobacillus-inoculated vacuum-packed rainbow trout (Oncorhynchus mykiss) fillets. J Agric Food Chem 2006, 54:4277-4283.

6. Vermeiren L, Devlieghere F, Debevere J: Evaluation of meat born lactic acid bacteria as protective cultures for biopreservation of cooked meat products. Int J Food Microbiol 2004, 96:149-164.

7. Chaillou S, Champomier-Vergès MC, Cornet M, Crutz-Le Coq AM, Dudez AM, Martin V, Beaufils S, Darbon-Rongere E, Bossy R, Loux V, Zagorec M: The complete genome sequence of the meat-borne lactic acid bacterium Lactobacillus sakei 23 K. Nat Biotechnol 2005, 23:1527-1533.

8. Lauret $R$, Morel-Deville F, Berthier F, Champomier-Vergès M, Postma $P$, Ehrlich SD, Zagorec M: Carbohydrate utilization in Lactobacillus sake. Appl Environ Microbiol 1996, 62:1922-1927.

9. McLeod A, Nyquist OL, Snipen L, Naterstad K, Axelsson L: Diversity of Lactobacillus sakei strains investigated by phenotypic and genotypic methods. Syst Appl Microbiol 2008, 31:393-403.

10. Chiaramonte F, Blugeon S, Chaillou S, Langella P, Zagorec M: Behavior of the meat-borne bacterium Lactobacillus sakei during its transit through the gastrointestinal tracts of axenic and conventional mice. App/ Environ Microbiol 2009, 75:4498-4505.

11. Dal Bello F, Walter J, Hammes WP, Hertel C: Increased complexity of the species composition of lactic acid bacteria in human feces revealed by alternative incubation condition. Microb Ecol 2003, 45:455-463.

12. Walker A, Cerdeno-Tarraga A, Bentley S: Faecal matters. Nat Rev Microbiol 2006, 4:572-573.

13. Chiaramonte F, Anglade P, Baraige F, Gratadoux JJ, Langella P, Champomier-Vergès MC, Zagorec M: Analysis of Lactobacillus sakei mutants selected after adaptation to the gastrointestinal tract of axenic mice. Appl Environ Microbiol 2010, 76:2932-2939.

14. Stentz R, Lauret R, Ehrlich SD, Morel-Deville F, Zagorec M: Molecular cloning and analysis of the ptsHI operon in Lactobacillus sake. Appl Environ Microbiol 1997, 63:2111-2116.

15. Stentz R, Cornet M, Chaillou S, Zagorec M: Adaption of Lactobacillus sakei to meat: a new regulatory mechanism of ribose utilization? INRA, EDP Sciences 2001, 81:131-138.

16. Stentz R, Zagorec M: Ribose utilization in Lactobacillus sakei: analysis of the regulation of the $r b s$ operon and putative involvement of a new transporter. J Mol Microbiol Biotechnol 1999, 1:165-173.

17. Torriani S, Clementi F, Vancanneyt M, Hoste B, Dellaglio F, Kersters K: Differentiation of Lactobacillus plantarum, $L$. pentosus and $L$. paraplantarum species by RAPD-PCR and AFLP. Syst Appl Microbiol 2001, 24:554-560.

18. Claesson MJ, van Sinderen D, OToole PW: The genus Lactobacillus - a genomic basis for understanding its diversity. FEMS Microbiol Lett 2007, 269:22-28.

19. McLeod A, Zagorec M, Champomier-Vergès MC, Naterstad K, Axelsson L: Primary metabolism in Lactobacillus sakei food isolates by proteomic analysis. BMC Microbiol 2010, 10:120.

20. Deutscher J, Francke C, Postma PW: How phosphotransferase systemrelated protein phosphorylation regulates carbohydrate metabolism in bacteria. Microbiol Mol Biol Rev 2006, 70:939-1031.

21. Stulke J, Hillen W: Carbon catabolite repression in bacteria. Curr Opin Microbiol 1999, 2:195-201

22. Titgemeyer F, Hillen W: Global control of sugar metabolism: a grampositive solution. Antonie Van Leeuwenhoek 2002, 82:59-71.

23. Fujita Y: Carbon catabolite control of the metabolic network in Bacillus subtilis. Biosci Biotechnol Biochem 2009, 73:245-259.

24. Schumacher MA, Allen GS, Diel M, Seidel G, Hillen W, Brennan RG: Structural basis for allosteric control of the transcription regulator $\mathrm{CcpA}$ by the phosphoprotein HPr-Ser46-P. Cell 2004, 118:731-741.

25. Obst M, Hehn R, Vogel RF, Hammes WP: Lactose metabolism in Lactobacillus curvatus and Lactobacillus sake. FEMS Microbiol Lett 1992, 97:209-214.

26. Montel MC, Champomier MC: Arginine catabolism in Lactobacillus sake isolated from meat. Appl Environ Microbiol 1987, 53:2683-2685.

27. Zuniga $M$, Champomier-Vergès $M$, Zagorec $M$, Pérez-Martinez G: Structural and functional analysis of the gene cluster encoding the enzymes of the arginine deiminase pathway of Lactobacillus sake. J Bacteriol 1998 180:4154-4159.

28. Rodionov DA, Mironov AA, Gelfand MS: Transcriptional regulation of pentose utilisation systems in the Bacillus/Clostridium group of bacteria. FEMS Microbiol Lett 2001, 205:305-314

29. Berthier F, Zagorec M, Champomier-Vergès MC, Ehrlich SD, Morel-Deville F: Efficient transformation of Lactobacillus sake by electroporation. Microbiol 1996, 142:1273-1279.

30. Hagen BF, Næs $H$, Holck AL: Meat starters have individual requirements for Mn2+. Meat Science 2000, 55:161-168.

31. Møretrø T, Hagen BF, Axelsson L: A new, completely defined medium for meat lactobacilli. J App/ Microbiol 1998, 85:715-722.

32. Nyquist OL, McLeod A, Brede DA, Snipen L, Nes IF: Comparative genomics of Lactobacillus sakei with emphasis on strains from meat. Mol Genet Genomics 2011, 285:297-311.

33. Rud I, Naterstad K, Bongers RS, Molenaar D, Kleerebezem M, Axelsson L: Functional analysis of the role of CggR (central glycolytic gene regulator) in Lactobacillus plantarum by transcriptome analysis. Microbial Biotechnology 2011, 4:345-356.

34. Vebø HC, Solheim M, Snipen L, Nes IF, Brede DA: Comparative genomic analysis of pathogenic and probiotic Enterococcus faecalis isolates, and 
their transcriptional responses to growth in human urine. PLoS One 2010, 5:e12489.

35. Smyth GK, Speed T: Normalization of cDNA microarray data. Methods 2003, 31:265-273.

36. Smyth GK: Linear models and empirical bayes methods for assessing differential expression in microarray experiments. Stat Appl Genet Mol Biol 2004, 3:Article3.

37. Smyth GK, Michaud J, Scott HS: Use of within-array replicate spots for assessing differential expression in microarray experiments. Bioinformatics 2005, 21:2067-2075.

38. Rode TM, Møretrø T, Langsrud S, Langsrud O, Vogt G, Holck A: Responses of Staphylococcus aureus exposed to $\mathrm{HCl}$ and organic acid stress. Can J Microbiol 2010, 56:777-792.

39. Weickert MJ, Chambliss GH: Site-directed mutagenesis of a catabolite repression operator sequence in Bacillus subtilis. Proc Natl Acad Sci USA 1990, 87:6238-6242.

40. Champomier-Vergès MC, Chaillou S, Cornet M, Zagorec M: Erratum to "Lactobacillus sakei: recent developments and future prospects". Res Microbiol 2002, 153:115-123.

41. Lorquet F, Goffin P, Muscariello L, Baudry JB, Ladero V, Sacco M, Kleerebezem M, Hols P: Characterization and functional analysis of the poxB gene, which encodes pyruvate oxidase in Lactobacillus plantarum. J Bacteriol 2004, 186:3749-3759.

42. Muscariello L, Marasco R, De Felice M, Sacco M: The functional ccpA gene is required for carbon catabolite repression in Lactobacillus plantarum. Appl Environ Microbiol 2001, 67:2903-2907.

43. Branny P, De La Torre F, Garel JR: Cloning, sequencing, and expression in Escherichia coli of the gene coding for phosphofructokinase in Lactobacillus bulgaricus. J Bacteriol 1993, 175:5344-5349.

44. Viana R, Perez-Martinez G, Deutscher J, Monedero V: The glycolytic genes pfk and pyk from Lactobacillus casei are induced by sugars transported by the phosphoenolpyruvate:sugar phosphotransferase system and repressed by CcpA. Arch Microbiol 2005, 183:385-393.

45. Kandler O: Carbohydrate metabolism in lactic acid bacteria. Antonie Van Leeuwenhoek 1983, 49:209-224.

46. Naterstad K, Rud I, Kvam I, Axelsson L: Characterisation of the gap operon from Lactobacillus plantarum and Lactobacillus sakei. Curr Microbiol 2007, 54:180-185.

47. Kim I, Kim E, Yoo S, Shin D, Min B, Song J, Park C: Ribose utilization with an excess of mutarotase causes cell death due to accumulation of methylglyoxal. J Bacteriol 2004, 186:7229-7235.

48. Weber J, Kayser A, Rinas U: Metabolic flux analysis of Escherichia coli in glucose-limited continuous culture. II. Dynamic response to famine and feast, activation of the methylglyoxal pathway and oscillatory behaviour. Microbiology 2005, 151:707-716.

49. Totemeyer S, Booth NA, Nichols WW, Dunbar B, Booth IR: From famine to feast: the role of methylglyoxal production in Escherichia coli. Mol Microbiol 1998, 27:553-562.

50. Malleret C, Lauret R, Ehrlich SD, Morel-Deville F, Zagorec M: Disruption of the sole IdhL gene in Lactobacillus sakei prevents the production of both L- and D-lactate. Microbiology 1998, 144:3327-3333.

51. Axelsson L: Lactic acid bacteria: classification and physiology. In Lactic acid bacteria: microbiological and functional aspects.. Third revised and expanded edition. Edited by: Salminen S, von Wright A, Ouwehand A. New York, USA: Marcel Dekker, Inc./CRC Press; 2004:1-66.

52. Condon S: Responses of lactic acid bacteria to oxygen. FEMS Microbiol Rev 1987, 46:269-280.

53. Fridovich I: The biology of oxygen radicals. Science 1978, 201:875-880.

54. Rodionov DA, Vitreschak AG, Mironov AA, Gelfand MS: Comparative genomics of thiamin biosynthesis in procaryotes. New genes and regulatory mechanisms. J Biol Chem 2002, 277:48949-48959.

55. Jordan A, Reichard P: Ribonucleotide reductases. Annu Rev Biochem 1998 67:71-98.

56. Keeney KM, Stuckey JA, O'Riordan MX: LpIA1-dependent utilization of host lipoyl peptides enables Listeria cytosolic growth and virulence. Mol Microbiol 2007, 66:758-770.

57. Marceau A, Zagorec M, Chaillou S, Mera T, Champomier-Vergès MC: Evidence for involvement of at least six proteins in adaptation of Lactobacillus sakei to cold temperatures and addition of $\mathrm{NaCl}$. Appl Environ Microbiol 2004, 70:7260-7268.
58. Grogan DW, Cronan JE Jr: Cyclopropane ring formation in membrane lipids of bacteria. Microbiol Mol Biol Rev 1997, 61:429-441.

59. Schujman GE, Guerin M, Buschiazzo A, Schaeffer F, Llarrull LI, Reh G, Vila AJ, Alzari PM, de Mendoza D: Structural basis of lipid biosynthesis regulation in Gram-positive bacteria. Embo J 2006, 25:4074-4083.

60. Mahr K, Hillen W, Titgemeyer F: Carbon catabolite repression in Lactobacillus pentosus: analysis of the ccpA region. Appl Environ Microbiol 2000, 66:277-283.

61. Nentwich SS, Brinkrolf K, Gaigalat L, Huser AT, Rey DA, Mohrbach T, Marin K, Puhler A, Tauch A, Kalinowski J: Characterization of the Lacltype transcriptional repressor RbsR controlling ribose transport in Corynebacterium glutamicum ATCC 13032. Microbiology 2009, 155:150-164.

62. Muller W, Horstmann N, Hillen W, Sticht $H$ : The transcription regulator RbsR represents a novel interaction partner of the phosphoprotein HPrSer46-P in Bacillus subtilis. Febs J 2006, 273:1251-1261.

63. Perez-Rueda E, Collado-Vides J: The repertoire of DNA-binding transcriptional regulators in Escherichia coli K-12. Nucleic Acids Res 2000, 28:1838-1847.

64. Brinkrolf K, Ploger S, Solle S, Brune I, Nentwich SS, Huser AT, Kalinowski J, Puhler A, Tauch A: The Lacl/GalR family transcriptional regulator UriR negatively controls uridine utilization of Corynebacterium glutamicum by binding to catabolite-responsive element (cre)-like sequences. Microbiology 2008, 154:1068-1081

65. Saxild HH, Andersen LN, Hammer K: dra-nupC-pdp operon of Bacillus subtilis: nucleotide sequence, induction by deoxyribonucleosides, and transcriptional regulation by the deoR-encoded DeoR repressor protein J Bacteriol 1996, 178:424-434.

66. Zeng $\mathrm{X}$, Saxild $\mathrm{HH}$ : Identification and characterization of a DeoR-specific operator sequence essential for induction of dra-nupC-pdp operon expression in Bacillus subtilis. J Bacteriol 1999, 181:1719-1727.

67. Zeng X, Saxild HH, Switzer RL: Purification and characterization of the DeoR repressor of Bacillus subtilis. J Bacteriol 2000, 182:1916-1922.

68. Schuch R, Garibian A, Saxild HH, Piggot PJ, Nygaard P: Nucleosides as a carbon source in Bacillus subtilis: characterization of the drm-pupG operon. Microbiology 1999, 145:2957-2966.

69. Posthuma CC, Bader R, Engelmann R, Postma PW, Hengstenberg W, Pouwels PH: Expression of the xylulose 5-phosphate phosphoketolase gene, $x p k A$, from Lactobacillus pentosus MD363 is induced by sugars that are fermented via the phosphoketolase pathway and is repressed by glucose mediated by $\mathrm{CcpA}$ and the mannose phosphoenolpyruvate phosphotransferase system. Appl Environ Microbiol 2002, 68:831-837.

70. Charrier V, Buckley E, Parsonage D, Galinier A, Darbon E, Jaquinod M, Forest E, Deutscher J, Claiborne A: Cloning and sequencing of two enterococcal glpK genes and regulation of the encoded glycerol kinases by phosphoenolpyruvate-dependent, phosphotransferase systemcatalyzed phosphorylation of a single histidyl residue. J Biol Chem 1997, 272:14166-14174.

71. Darbon E, Servant P, Poncet S, Deutscher J: Antitermination by GlpP, catabolite repression via CcpA and inducer exclusion triggered by $\mathrm{P}$ GlpK dephosphorylation control Bacillus subtilis glpFK expression. Mol Microbiol 2002, 43:1039-1052.

72. Barrangou R, Azcarate-Peril MA, Duong T, Conners SB, Kelly RM, Klaenhammer TR: Global analysis of carbohydrate utilization by Lactobacillus acidophilus using cDNA microarrays. Proc Natl Acad Sci USA 2006, 103:3816-3821.

73. Chaillou S, Postma PW, Pouwels PH: Contribution of the phosphoenolpyruvate:mannose phosphotransferase system to carbon catabolite repression in Lactobacillus pentosus. Microbiology 2001, 147:671-679.

74. Veyrat A, Gosalbes MJ, Perez-Martinez G: Lactobacillus curvatus has a glucose transport system homologous to the mannose family of phosphoenolpyruvate-dependent phosphotransferase systems. Microbiology 1996, 142:3469-3477.

75. Veyrat A, Monedero V, Perez-Martinez G: Glucose transport by the phosphoenolpyruvate:mannose phosphotransferase system in Lactobacillus casei ATCC 393 and its role in carbon catabolite repression. Microbiology 1994, 140:1141-1149.

76. Viana R, Monedero V, Dossonnet V, Vadeboncoeur C, Perez-Martinez G, Deutscher J: Enzyme I and HPr from Lactobacillus casei: their role in 
sugar transport, carbon catabolite repression and inducer exclusion. $\mathrm{Mol}$ Microbiol 2000, 36:570-584.

doi:10.1186/1471-2180-11-145

Cite this article as: McLeod et al:: Global transcriptome response in

Lactobacillus sakei during growth on ribose. BMC Microbiology 2011

11:145.

Submit your next manuscript to BioMed Central and take full advantage of:

- Convenient online submission

- Thorough peer review

- No space constraints or color figure charges

- Immediate publication on acceptance

- Inclusion in PubMed, CAS, Scopus and Google Scholar

- Research which is freely available for redistribution

Submit your manuscript at 